\title{
1 Blastocyst trophectoderm endocytic activation, a marker of adverse
}

\section{2 developmental programming}

3

4

$5 \quad$ Laura Caetano ${ }^{1}$, Judith J. Eckert ${ }^{2}$, David Johnston ${ }^{3}$, David S. Chatelet ${ }^{3}$, David A.

6 Tumbarello ${ }^{4}$, Neil R. Smyth ${ }^{1}$, Sue Ingamells ${ }^{5}$, Anthony Price ${ }^{5}$ and Tom P. Fleming ${ }^{1}$ * 7

$8 \quad{ }^{1}$ Biological Sciences, Southampton General Hospital, University of Southampton,

9 Southampton SO16 6YD, UK.

$10{ }^{2}$ Human Development and Health, Southampton General Hospital, University of

11 Southampton, Southampton SO16 6YD, UK

$12{ }^{3}$ Biomedical Imaging Unit, Southampton General Hospital, University of Southampton,

13 Southampton SO16 6YD, UK

$14{ }^{4}$ Biological Sciences, Life Sciences Building 85, University of Southampton, Southampton $15 S O 171 B J, U K$

$16 \quad{ }^{5}$ Wessex Fertility Clinic, Southampton SO15 5QS

17

*Correspondence address. Tel: +44-7833 048158; E-mail: t.p.fleming@,soton.ac.uk

20 Short title: Blastocyst trophectoderm endocytic activation

21 Keywords: Trophectoderm, mouse blastocyst, human blastocyst, endocytosis, lysosomes, 22 TFEB

23 Words: 6405 (excluding references and figure legends and tables) 


\section{Abstract}

The mouse preimplantation embryo is sensitive to its environment including maternal dietary protein restriction which can alter the developmental programme and affect lifetime health. Previously, we have shown maternal low protein diet (LPD) causes reduction in blastocyst $\mathrm{mTORC} 1$ signalling coinciding with reduced availability of branched-chain amino acids (BCAAs) in surrounding uterine fluid. BCAA deficiency leads to increased endocytosis and lysosome biogenesis in blastocyst trophectoderm (TE), a response to promote compensatory histotrophic nutrition. Here, we first investigated the induction mechanism by individual variation in BCAA deficiency in an in vitro quantitative model of TE responsiveness. We found isoleucine (ILE) deficiency as the most effective activator of TE endocytosis and lysosome biogenesis, with less potent roles for other BCAAs and insulin; cell volume was also influential. TE response to low ILE included upregulation of vesicles comprising megalin receptor and cathepsin-B and the response was activated from blastocyst formation. Second, we identified the transcription factor TFEB as mediating the histotrophic response by translocation from cytoplasm to nucleus during ILE deficiency and in response to mTORC1 inhibition. Lastly, we investigated whether a similar mechanism responsive to maternal nutritional status was found in human blastocysts. Blastocysts from women with high body-mass index, but not the method of fertilisation, revealed stimulated lysosome biogenesis and TFEB nuclear migration. We propose TE lysosomal phenotype as an early biomarker of environmental nutrient stress that may associate with longterm health outcome. 


\section{Introduction}

49 The preimplantation embryo can sense the levels of maternal tract nutrients in vivo and adjust its phenotype and its developmental programme to match these conditions and so aid survival. Thus, maternal dietary protein restriction induces the mouse blastocyst to upregulate trophectoderm proliferation, endocytosis, and subsequent cellular motility and invasiveness at implantation, leading to increased placental efficiency, a combination of adaptations protecting development and offspring competitiveness (Coan, et al. 2011, Eckert, et al. 2012, Sun, et al. 2014, Watkins, et al. 2015). However, whilst nutrient sensing derived from poor maternal diet may activate early compensatory responses, these also associate with later life chronic disease risk of offspring across impaired growth, cardiometabolic and neurological morbidities (Gould, et al. 2018, Lanham, et al. 2020, Watkins, et al. 2011, Watkins, et al. 2008). A similar sensitivity has been identified in the human preimplantation embryo with respect to culture environment and assisted reproductive treatment (ART) affecting development and postnatal growth and disease risk (Feuer and Rinaudo 2016, Kleijkers, et al. 2016, Sunde, et al. 2016). Thus, the early mammalian embryo is recognised as a vulnerable developmental stage in the concept known as 'Developmental Origin of Health and Disease' (DOHaD) (Fleming, et al. 2018, Hanson and Gluckman 2014). Our previous work has concerned the induction of nutrient sensing in the mouse blastocyst given its importance across lifetime health. We found maternal low protein diet (LPD) from conception caused branched-chain amino acid (BCAA) levels (leucine, LEU; isoleucine, ILE; valine, VAL) to become depleted within the uterine fluid at the time of blastocyst formation; this coincided with reduced insulin concentration in maternal serum, all before implantation occurred (Eckert, et al. 2012). The dietary decrease in metabolites resulted in a reduction in blastocyst mammalian target of rapamycin complex 1 (mTORC1) 


\section{Materials and Methods}

\section{Animals and embryo collection}

growth-regulating signalling (Takahara, et al. 2020) through the S6-kinase pathway (Eckert, et al. 2012) and an increase in trophectoderm (TE) endocytosis and lysosome biogenesis mediated through cytoskeletal reorganisation regulated by Rho-GTPase (Sun, et al. 2014). The endocytosis response likely represents increased histotrophic nutrition of tract proteins and lipids to combat poor maternal nutrition. Moreover, the endocytic response could be induced in vitro by culture of control embryos in medium with deficient albumin or, more specifically, deficient in BCAAs to mimic the LPD uterine fluid but with all other amino acids at levels found in normal-fed dams (NPD) (Sun, et al. 2014). More extensive manipulations of mouse embryo culture environment have demonstrated the combination of low BCAAs with low insulin levels were sufficient to induce the altered growth and cardiovascular disease phenotype in later life (Velazquez, et al. 2018).

In the current study, we further analyse the environmental responsiveness of mouse TE to identify more precisely the extracellular conditions required to induce enhanced endocytosis and lysosome biogenesis, its timing of induction during cleavage, and the mechanism regulating the cytoplasmic restructuring involved. We also conducted a preliminary investigation of whether a similar TE endocytic/lysosomal phenotype occurs in the human blastocyst (donated to research from ART) with respect to maternal metabolic condition. Collectively, our data provides new insight into the conditions causing adverse reprogramming of early development with biological and clinical implications.

MF1 outbred mice, kept under UK Home Office Project license and local ethics approval, were bred in-house (University of Southampton Biomedical Research Facility) in accordance 
with the Animals (Scientific Procedures) Act of 1986 and associated Codes of Practise on a 07:00-19:00 h light cycle fed with standard chow. Virgin females (7-9 weeks) were naturally mated overnight with MF1 males (2-6 months) and plug positive females were housed individually the following morning. Mated dams were at E0.5 at $14.00 \mathrm{~h}$ on the day the vaginal plug was detected. Two-cell embryos were flushed from the oviducts at E1.5 with H6 medium with $4 \mathrm{mg} / \mathrm{ml}$ BSA (H6+BSA) (Watkins, et al. 2007) after cervical dislocation and dissection of the reproductive tract.

\section{In vitro culture}

Collected 2-cell embryos were pooled and allocated to culture medium to the blastocyst stage to investigate quantitatively which components of the depleted BCAA and insulin composition found in LPD dams were most effective in inducing the enhanced endocytosis and lysosome biogenesis phenotype found in LPD blastocysts (Eckert et al, 2012; Sun et al, 2014). Embryos at the 2-cell stage were cultured in defined potassium simplex optimized medium (KSOM medium) in the absence of BSA but with variable amino acid (AA) composition (Velazquez, et al. 2018), checked for osmolarity (250-260 mOsm) and used under mineral oil at $37^{\circ} \mathrm{C}$ in $5 \% \mathrm{CO}_{2}$ until the morula or blastocyst stage of development, depending on experimental design. Control medium consisted of KSOM supplemented with insulin $(1 \mathrm{ng} / \mathrm{ml})$ (ThermoFisher) and the complete amino acid composition as previously found in the MF1 uterine luminal fluid of dams at E3.5 fed a normal protein diet (Table 1) (Eckert, et al. 2012). This included the BCAAs valine $(0.46 \mathrm{mM})$, isoleucine $(0.21 \mathrm{mM})$ and leucine $(0.32 \mathrm{mM})$. In experimental treatment groups, a combination or individual BCAA concentration was decreased (50\%, L-) compared with control (100\%, N-BCAA) whilst all other AA concentrations remained unchanged. Also, insulin was included at normal (N-INS, $1 \mathrm{ng} / \mathrm{ml}$ ) or decreased by 50\% (L-INS). All AAs were sourced from Sigma. 
124 Endocytosis assay

125 After culture, embryos at E3.5 had reached the blastocyst stage and were incubated for 1 hour 126 in the same medium but containing Self-Quenched BODIPY FL Conjugate of BSA (BSA-

127 BODIPY, $0.5 \mathrm{mg} / \mathrm{ml}$ ) (BioVision) and LysoTracker Red DND-99 (100 nM) (ThermoFisher)

128 or Magic Red ${ }^{\mathrm{TM}}$ (Bio-Rad) to label degraded protein after endocytosis, lysosomes and

129 Cathepsin B, respectively. After incubation, embryos were washed 3 times in H6+BSA and

130 fixed in 1\% formaldehyde in PBS for 20 minutes. After fixation, embryos were also washed

131 in PBS 3 times and labelled with the plasma membrane stain CellMask ${ }^{\mathrm{TM}}$ Deep Red (Thermo

132 Fisher Scientific) for $1 \mathrm{~h}$ at room temperature (1:200 in PBS) to label trophectoderm (TE) cell

133 boundaries. At that stage, embryos were either stained with $0.2 \mu \mathrm{g} / \mathrm{ml}$ DAPI (Invitrogen),

134 washed 3 times in Tween-20 (Sigma-Aldrich) 1:1000 in PBS (PBS-T) and mounted with 20

$135 \mu$ l Citifluor or underwent immunocytochemistry.

136

137 Rapamycin treatment

138 2-cell stage embryos (E1.5) were cultured after collection in N-BCAA/N-INS medium

139 (Table 1) supplemented with rapamycin (LC laboratories, Woburn, USA) at final

140 concentrations of $100 \mathrm{nM}, 1 \mu \mathrm{M}$ or $20 \mu \mathrm{M}$ at $37^{\circ} \mathrm{C}$ in $5 \% \mathrm{CO}_{2}$. After 36 hours in culture,

141 embryos were transferred to fresh medium containing the same concentration of rapamycin

142 until reaching the blastocyst stage. Control embryos were cultured in KSOM without

143 rapamycin but complemented with DMSO at 1:1000 (rapamycin solvent). At that point,

144 embryos were stained with LysoTracker, fixed in 1\% formaldehyde in PBS for 20 minutes

145 and imaged by confocal microscopy before immunocytochemistry.

\section{Immunocytochemistry}


148 Antibodies used for immunolabelling were: rabbit polyclonal anti transcription factor EB

149 (TFEB) gene 7942 (A303-673A-T Bethyl Laboratories; 1:50); mouse monoclonal to megalin

150 (Protein G purified, 1:400) (Meads and Wild 1993); and rabbit polyclonal anti-clathrin (Cell

151 Signaling P1663, 1:400). Secondary antibodies were: Alexa 546, 488 and 633 (Invitrogen;

152 1:300). Negative controls were included by omitting the primary antibody. Embryos,

153 previously fixed and stained with CellMask, were subsequently permeabilised with $0.25 \%$

154 Triton-X-100 (Sigma-Aldrich) in PBS (PBS-T) for 15 minutes, washed in PBS-T and

155 neutralised in $2.6 \mathrm{mg} / \mathrm{ml} \mathrm{NH}_{4} \mathrm{Cl}$ (Sigma-Aldrich) in PBS for 10 minutes. Embryos were then

156 washed 3 times (5 minutes each) in PBS-T before incubation with primary antibody (diluted

157 in PBS-T) overnight at $4^{\circ} \mathrm{C}$. The following day, embryos were washed with PBS-T (3 times

158 for 10 minutes each), incubated with secondary antibody for $1 \mathrm{~h}$ at room temperature, washed

159 in PBS-T (3 times for 10 minutes), nuclear stained with DAPI (Invitrogen; $0.2 \mu \mathrm{g} / \mathrm{ml}$ in PBS-

160 T; 20 mins), washed 3 times in PBS-T and mounted with $20 \mu 1$ Citifluor.

161

162 Confocal microscopy, image capture and analysis

163 Embryos were viewed with a Leica SP5 confocal microscope. Images were acquired by

164 accumulation of $z$-series of TE cells on the surface closest to the coverslip, 50-60 xy sections

165 at $0.15 \mu \mathrm{m}$ intervals, tangential to the embryo surface and extending from apical to basal

166 surfaces of examined TE cells to provide a high resolution dataset. Images from confocal

167 microscopy were analysed with VOLOCITY-3D 6.3 quantification software (PerkinElmer).

168 Measurements were made in individual TE cells with 1-3 adjacent TE cells analysed per

169 embryo. A VOLOCITY protocol was designed for each individual labelling method used

170 based on vesicle/structure sizes and applied to all embryos in different treatment groups. The

171 optimised protocol is provided in Supplementary Table 1. Using these settings,

172 VOLOCITY was used to calculate number and volume of labelled vesicles per TE cell and to 
173 measure the distance from the vesicles centre to the nucleus edge. VOLOCITY was also used

174 to measure cell volume as required.

\section{Human embryo study}

177 Vitrified embryos were donated with full patient consent for research under HFEA licence

178 from Wessex Fertility Clinic, Southampton. Donors were selected based upon maternal BMI

179 (two groups, normal 18.9-22.9; high $>25$; 27.1-32.3); patient age (less than 38); embryo

180 number per patient (minimum 3); embryo stage (all vitrified from day (D) 3 or later). The

181 normal and high BMI patient groups and embryos analysed are shown in Table 2. Vitrified

182 embryos were thawed with Vit Kit®-Thaw 90137-SO (Irvine Scientific) for 5 minutes at

$18337^{\circ} \mathrm{C}$ in $40 \mu \mathrm{l}$ drop of thawing solution in a 4-well dish under sterile conditions on heated

184 stage. Embryos were then transferred to the kit's dilution solution at room temperature for 4

185 minutes followed by $2 \times 4$-minute washes in washing solution at room temperature. Embryos

186 were then transferred singly into pre-equilibrated drops $(40 \mu \mathrm{l})$ of Sage 1-step ${ }^{\mathrm{TM}}$ medium

187 with HSA and phenol red (Origio) in a $6 \mathrm{~cm}$ dish covered with mineral oil (Origio) and

188 incubated at $37^{\circ} \mathrm{C}$ with $5 \% \mathrm{CO}_{2}$ and $5 \% \mathrm{O}_{2}$ to develop to blastocyst stage at D5. At this

189 stage, embryos were incubated with LysoTracker (as used for mouse embryos but omitting

190 BSA-BODIPY co-labelling), fixed and immunolabelled with TFEB (as for mouse embryos).

191

\section{Statistical analysis}

193 Mouse embryo data were first assessed for normality using the Shapiro-Wilk normality test.

194 Data analysis was performed using One-Way ANOVA and Tukey's post hoc test for multi

195 comparisons or Student's t-test for normally distributed data ( 75\% samples); and with

196 Kruskal-Wallis test with Dunn's multiple comparisons post hoc test or Mann-Whitney for

197 non-normally distributed data ( $25 \%$ samples). Pooling data across experiments to increase 
sample number showed lysosome number per cell to be normally distributed, and analysis of our non-normalised data by tests for normalised data did not alter statistical significance.

200 Human embryo lysosome biogenesis and immunocytochemistry data were assessed using the 201 multilevel random effects regression model (SPSS version 25) which takes into account

202 potential hierarchical nature of the data with between-patient and within-patient variation and 203 different parameters measured from individual embryos. Thus, differences identified between groups studied are independent of cell volume, cell number and whether a resultant pregnancy occurred from a sibling embryo collected at the same time. Data are presented as dot plots and, for mouse, with box and whisker markers to identify the median and interquartile range and $\mathrm{min} / \mathrm{max}$ points with $\mathrm{P}<0.05$ regarded as significant.

\section{Results}

211 A role for isoleucine in activating mouse embryo endocytosis and lysosome biogenesis

212 Our first experiments were to identify the relative role of deficiency of the three BCAAs,

213 leucine (LEU), isoleucine (ILE) and valine (VAL), shown previously to enhance endocytosis 214 and lysosome biogenesis in trophectoderm (TE) in our diet and in vitro models (Eckert, et al. 215 2012, Sun, et al. 2014) and whether deficient insulin was contributory. Control 2-cell

216 embryos were cultured to blastocyst stage ( $\sim 8 \mathrm{~h})$ in KSOM medium with the complete

217 uterine fluid composition of AAs (Table 1) and systemic insulin (1 ng/ml) as found in NPD-

218 fed dams (N-BCAA/N-INS). In tested culture medium, one of three BCAAs was individually

219 reduced by 50\% compared to the NPD uterine fluid concentration and normal insulin (L-

220 VAL/N-INS; L-LEU/N-INS; L-ILE/N-INS), or normal concentration of BCAAs but 50\%

221 decreased insulin (N-BCAA/L-INS). Blastocysts were examined for endocytosis and 
lysosomes using combined BSA-BODIPY and LysoTracker assay by confocal microscopy and image analysis.

In all groups, LysoTracker and BSA-BODIPY labelled vesicles were mainly colocalised around the TE nucleus, viewed en face, tangential to the coverslip (Figure 1A) with data accrued from high resolution z-series across the complete cell layer. Blastocysts in the L-ILE/N-INS group had increased number and collective volume of BSA-BODIPY positive vesicles per cell ( $\mathrm{P}=0.04,0.03$ respectively) while LysoTracker number and collective volume per cell were increased but not significantly ( $\mathrm{P}=0.09$ and 0.06 , respectively) (Figure 1B-E) compared to the control N-BCAA/N-INS. L-ILE/N-INS was also increased in BSABODIPY vesicle number compared to L-VAL/N-INS $(\mathrm{P}=0.017)$ and N-BCAA/L-INS $(\mathrm{P}=0.014)$ and in collective volume of vesicles in N-BCAA/L-INS P=0.05) (Figure 1C,E). L-ILE/N-INS LysoTracker vesicle number and collective volume was increased compared with N-BCAA/L-INS P=0.02 and 0.02 respectively) (Figure 1B,D). In contrast, L-VAL/NINS, L-LEU/N-INS and N-BCAA/L-INS were not different in vesicle labelling from the NBCAA/N-INS controls although L-LEU/N-INS mean or median values for all analyses were above those of other groups except L-ILE/N-INS, a larger dataset would be required to expose any effect (Figure 1B-D). Further analysis showed that the increase in L-ILE/N-INS vesicle dynamics was not caused by increased vesicle size compared to controls indicating endocytosis and lysosome enhancement were by collective increase in vesicle number (Supplemental Figure 1A,B). The average distance of both vesicles (BSA-BODIPY and LysoTracker) to the nucleus was not altered across treatments (Supplemental Figure 1C,D). Lastly, all treatments resulted in a consistent blastocyst formation rate of $\sim 70 \%$ and with equivalent cell numbers present (Supplemental Figure 1E,F) indicating these treatments did not affect embryo viability, as found for later treatments. Collectively, these data indicate 
embryo endocytosis and lysosomes were increased in response to reduced isoleucine (L-ILE)

248 alone in the culture medium, a partial response may be induced by low leucine (L-LEU),

249 while valine and insulin deficiency produced no effect.

To determine whether increased LysoTracker vesicle number and collective volume per cell in the L-ILE/N-INS group reflected an actual increase in lysosomal enzyme activity, blastocysts from this treatment were also assayed with Magic Red staining which fluoresces in response to cleavage by the lysosome enzyme, Cathepsin B (Figure 2A). In the L-ILE/NINS group, Magic Red staining was increased in terms of collective vesicle volume per TE cell $(\mathrm{P}=0.04)$ but the increase for vesicle number per cell was not significant $(\mathrm{P}=0.1)$ compared to the N-BCAA/N-INS control (Figure 2B,C).

To investigate whether endocytosis upregulation in response to L-ILE/N-INS coincided with increased expression of endocytic receptor, megalin, and endocytic vesicle coat protein, clathrin, these proteins were analysed by immunocytochemistry (Figure 2D-G). Both megalin and clathrin were concentrated along the apical surface of TE cells as well as within vesicles in the apical cytoplasm (Figure 2D,E). Megalin $(\mathrm{P}=0.02)$ and clathrin $(\mathrm{P}=0.002)$ signal intensities were increased in L-ILE/N-INS blastocyst TE cells when compared to the N-BCAA/N-INS control using standardised settings for fluorescent intensity by total voxel count per channel (Figure 2F,G).

\section{Combinations of depleted BCAAs and/or insulin can activate embryo endocytosis}

To further examine individual and combined BCAA interactions with insulin, embryos were collected at E1.5 (2-cell stage) and cultured until blastocyst stage ( $48 \mathrm{~h})$ in six KSOM media treatments: N-BCAA/N-INS (control), L-ILE/N-INS (positive control from above), L-ILE/L- 
INS, L-LEU/L-INS, L-ILE/L-LEU/L-INS and L-BCAA/L-INS (Figure 3A). An increase in BSA-BODIPY collective vesicle volume per cell $(\mathrm{P}=0.04)$ and vesicle number per cell $(\mathrm{P}=0.07)$ was present in the L-ILE/N-INS positive control. Increased BSA-BODIPY vesicle number and collective vesicle volume per cell were also present in the L-ILE/L-LEU/L-INS combination treatment ( $\mathrm{P}=0.002$ and 0.005 respectively) (Figure 3B-E). Collective volume of BSA-BODIPY vesicles per cell was higher but not significant in the L-BCAA/L-INS group $(\mathrm{P}=0.09)$. Other L-INS groups show a consistent non-significant increase in mean BSA-BODIPY vesicle number and collective volume per cell compared with the NBCAA/N-INS control. Similarly, LysoTracker vesicle number and collective volume per cell were increased in all combination treatments compared with control N-BCAA/N-INS but not to statistical significance (Figure 3B-E). In all six culture treatments, individual size of vesicles did not differ (Supplemental Figure 2A,B) indicating changes in collective volume where present reflected an increase in vesicle number. The mean distance of both vesicles to the nucleus was also not altered by treatment (Supplemental Figure 2C,D). Collectively, these data suggest minor stimulatory effects on endocytosis and lysosomes may be mediated by some combined metabolite combinations. The effect of L-ILE deficiency alone was the focus of future mouse experiments.

\section{Stimulation of lysosome biogenesis by low isoleucine activates at the blastocyst stage}

291 Embryos were collected at E1.5 (2-cell stage) and cultured until the morula stage (36 h; 16cell stage) in KSOM medium with L-ILE/N-INS or in control medium (N-BCAA/N-INS). At the morula stage, embryos were examined using the LysoTracker assay alongside blastocysts (cultured for $48 \mathrm{~h}$ ) used as a positive control (Figure 4A). Embryos cultured until the morula stage in L-ILE/N-INS group had no change in LysoTracker vesicle number or collective volume per cell compared to the N-BCAA/N-INS control group while blastocyst lysosome 
number $(\mathrm{P}=0.002)$ or collective volume $(\mathrm{P}=0.06)$ were enhanced (Figure 4B-E). Note, morula vesicle number/cell is about double that of the blastocyst reflecting larger cell volume at the morula 16-cell stage. Collectively, these data indicate that the capacity for lysosome

300 biogenesis initiates at the blastocyst stage.

Isoleucine deficiency activates TFEB nuclear translocation in blastocyst TE cells

We next considered further downstream regulation of enhanced endocytosis and lysosome biogenesis following induction by isoleucine deficiency. The transcription factor TFEB has been shown to interact with mTORC1 on lysosomal membranes and upon reduction in mTORC1 signalling is translocated to the nucleus and increases transcription of multiple genes implicated in lysosomal biosynthesis and autophagy (Sardiello, et al. 2009, Settembre, et al. 2012). TFEB subcellular localization was analysed in blastocysts after culture from 2cell stage in either L-ILE/N-INS or N-BCAA/N-INS (Figure 5A). To quantify cellular

310 localisation, the relative volumes of TFEB in the cytoplasm and nucleus in TE cells were

311 ratioed. TFEB switched from an approximate equal cytoplasmic / nuclear $(\sim 50: 50 \%)$

312 localisation in N-BCAA/N-INS to a predominant nuclear localisation ( $20: 80$ cytoplasmic / nuclear) $(\mathrm{P}=0.02)$ in L-ILE/N-INS culture (Figure 5B,C). Pearson Correlation was used with

314 VOLOCITY software to calculate the relative association between green (TFEB) and blue

315 (DAPI) channels and showed TFEB was localised more in the nucleus in L-ILE/N-INS

$316(\mathrm{P}=0.02)$ compared to N-BCAA/N-INS (Figure 5D).

\section{TFEB localisation in blastocysts is sensitive to mTORC1}

319 Embryos were collected at E1.5 (2-cell stage) and cultured in control N-BCAA/N-INS

320 medium supplemented with rapamycin to inhibit mTORC1 signalling at different 
assessed for TFEB localisation (Figure 6A) as previously described or were analysed in the same medium using the LysoTracker assay (Figure 7). Rapamycin $(20 \mu \mathrm{M})$ caused a

324 dramatic translocation of TFEB from cytoplasm to nucleus $(\mathrm{P}=0.0001)$ confirmed using

325 Pearson Correlation analysis $(\mathrm{P}=0.0001)$ with an intermediate effect evident at lower dose ( 1 $\mu \mathrm{M} ; \mathrm{P}=0.04$ ) (Figure 6B,C). However, no significant difference was found in LysoTracker staining between the three groups although an increase in collective volume of lysosomes was detected at $20 \mu \mathrm{M}$ rapamycin $(\mathrm{P}=0.06)$ (Figure 7B). These data first confirm that TFEB nuclear localisation in our model is regulated by loss of mTORC1 signalling, and there is evidence that mTORC1 may promote increased lysosome biogenesis.

\section{Effect of cell volume on endocytosis and lysosome biogenesis}

333 To understand whether the increase in endocytosis and lysosome biogenesis following LILE/N-INS culture was superimposed upon more general cellular mechanism(s) affecting the number or density of these organelles in relation to the cell cycle and division (Carlton, et al. 2020), the VOLOCITY software was used to measure cell volume using the same samples as reported above. No difference was found in blastocyst TE cell volume in relation to control and experimental treatments with deficient individual BCAAs or INS (Figure 8A). The relationship between cell volume and BSA-BODIPY or LysoTracker vesicle numbers was further explored through correlation analysis following control (N-BCAA/N-INS) and LILE/N-INS treatments. LysoTracker vesicle number and collective volume per cell in both treatments were positively correlated with cell volume (N-BCAA/N-INS P=0.0004 and

$343 \mathrm{P}=0.0006$ respectively; L-ILE/N-INS P=0.02 for both) (Figure 8B,C). However, BSA-

344 BODIPY vesicle number and collective volume per cell were only significantly positively correlated with cell volume in the L-ILE/N-INS group ( $\mathrm{P}=0.01$ and $\mathrm{P}=0.0009$ respectively)

346 (Figure 8D,E). Notably, the data indicate that the L-ILE/N-INS sample points and best-fit 
347 lines (red) lie above those for N-BCAA/N-INS (blue) indicating L-ILE/N-INS to be

348 stimulatory independent of cell volume. However, some L-ILE/N-INS data points show both

349 high vesicle number or volume combined with high cell volume indicating these factors can

350 be associated.

351

352

353

354

355

356

357

358

359

360

361

362

363

364

365

366

367

368

369

370

371

The N-BCAA/N-INS and L-ILE/N-INS treatments did not affect cell volume in those samples analysed for TFEB localisation (Figure 8F) but here, following correlation analysis, no correlation between TFEB \% nuclear staining and cell volume was apparent (Figure 8G). Collectively, these results indicate first that while cell volume is similar across treatments, it nevertheless has a general treatment-independent positive effect upon endocytosis and lysosomes. Second, L-ILE/N-INS treatment can enhance endocytosis and lysosome biogenesis both independent of cell volume but also in association with it.

\section{Activation of lysosome biogenesis in human embryos}

Embryos donated from 14 patients (7 in both normal and high BMI groups) were cultured ( $\mathrm{n}=81$ embryos, 42 in the normal BMI group, 39 in the high BMI group) from embryonic D3 ( $\sim$-cells) to D5 (blastocyst stage). From these, 36\% and 41\%, respectively, developed to blastocysts and had similar total embryo volume ( $\mathrm{P}=0.88$; data not shown) (Table 2). Thirteen blastocysts from 7 patients from the normal BMI group and 15 blastocysts from 7 patients from the high BMI group (3 embryos lost during processing) were analysed successfully using LysoTracker assay for lysosomes (Figure 9A,B) and subsequently for TFEB localisation using immunocytochemistry (Figure 10). Embryos from within the high BMI group exhibited an enhanced lysosome collective volume per cell $(\mathrm{P}=0.015)$, similar to that previously seen in mouse embryos, although individual embryo scores were highly variable in the high BMI group (Figure 9A,B). Also, embryo cell number and cell volume 
were not significantly different between the BMI groups (Figure 9C,D) although both were influencing cofactors in the lysosome statistical analysis whilst whether patient pregnancy occurred that cycle was not a cofactor. Furthermore, examination of human embryos for TFEB localisation (Figure 10A,B) revealed mean nuclear versus cytoplasmic localization in the high BMI group to be higher $(\mathrm{P}=0.1)$ (Figure 10B).

The LysoTracker and TFEB results shown in the scatter plots for BMI groups also include mother number for each embryo and reveal similarity in outcome between sibling embryos from the same mother (Figure 9B, 10B). In addition to BMI, the dataset was also screened for whether intra-cytoplasmic sperm injection (ICSI) had been employed or not. This revealed no effect on lysosome or TFEB localisation but further show the closeness in outcomes for sibling embryos (Figure 10C,D). Collectively, these data indicate lysosome activity and TFEB localisation in human blastocysts is sensitive to environmental factors including patient BMI.

\section{Discussion}

Our study centred on the functioning of the extra-embryonic trophectoderm (TE) layer, the first cell type to differentiate on the surface of the mammalian embryo. TE contributes to blastocyst morphogenesis through the timing of transepithelial transport which permits blastocoel formation and regulated nutrient and metabolite provision for the inner cell mass (ICM) comprising the entire stem cell pool for fetal development (Eckert and Fleming 2008).

Nutrient provision for the embryo is also mediated by histotrophic means through maturation of a polarised apicobasal endocytic pathway with fluid-phase and receptor-mediated processing via multi-ligand megalin and cubilin receptors for internalisation of uterine fluid 
397 proteins and lipids (Assémat, et al. 2005, Fleming and Pickering 1985, Kelleher, et al. 2019).

398 After implantation, the TE continues its supportive function and gives rise to the chorio-

399 allantoic placenta regulating the nutritional demands of the fetus throughout gestation. In this

400 context, the TE requires cellular mechanisms to ascertain environment nutrient levels to

401 sustain embryo and fetal growth through pregnancy despite variations in maternal lifestyle

402 and diet.

403

404

Past studies using the mouse LPD model have revealed maternal LPD reduced the concentration of BCAAs within maternal serum and the uterine fluid that bathes the morula and blastocyst before implantation, coinciding with reduced systemic insulin and a decrease in blastocyst mTORC1 serine/threonine kinase signalling through reduced phosphorylation of the downstream effector, ribosomal S6 protein (Eckert, et al. 2012). TE cells within LPD blastocysts respond by increased endocytosis and lysosome formation, which we considered a histotrophic response to compensate for poor maternal nutrition (Sun, et al. 2014). TE endocytosis is known to be insulin-sensitive (Dunglison, et al. 1995) and the increased endocytosis phenotype after LPD can be mimicked by culture of control blastocysts in medium deficient in either protein or the BCAAs (LEU, ILE, VAL) (Sun, et al. 2014). A similar histotrophic mechanism is found in ovine and porcine embryos whereby uterine fluid LEU and other amino acids signal through mTORC1 in TE cells to coordinate conceptus growth (Kim, et al. 2013, Kim, et al. 2011). Moreover, increased endocytosis is a recognised

417 cellular process outside developmental models to combat protein deprivation signalled

418 through low BCAA availability and mTORC1 (Jones, et al. 2012). 
biomarker for adverse developmental programming as LPD offspring in later life develop growth, cardiometabolic and neurological morbidities (Gould, et al. 2018, Lanham, et al. 2020, Watkins, et al. 2011, Watkins, et al. 2008), outcomes that can be induced in control embryos by culture in medium deficient in BCAA and insulin (Velazquez, et al. 2018). We consider the activation of histotrophic nutrition, along with other compensatory responses by extra-embryonic lineages to promote maternal nutrient delivery following LPD, despite protecting survival, to contribute to over-nutrition during fetal growth and a postnatal metabolism that encourages adiposity and chronic disease (discussed in (Fleming, et al. 2018, Velazquez, et al. 2019). Thus, the compensatory promotion of nutrient delivery activated by maternal LPD during preimplantation is maintained even if restricted diet is not continued beyond implantation leading to increased fetal : placental weight ratio in late gestation, offspring perinatal weight becoming positively correlated with later life disease risk, and, in female offspring, sustained overweight throughout life (Watkins, et al. 2015, Watkins, et al. 2011, Watkins, et al. 2008). The mechanisms underlying induction of the endocytosis and lysosome phenotype therefore acquire further importance in the search for protection measures against periconceptional DOHaD programming (Fleming, et al. 2018). endocytosis and lysosome formation in mouse blastocysts in vitro following culture in medium with amino acid composition matching that found within uterine fluid of control fed

442 (NPD) dams but with deficiency in BCAAs (Sun, et al. 2014). However, we have improved the protocol by omitting the need for zona removal by transient acidic medium which may cause cellular stress to embryos, and by refining the VOLOCITY image analysis of confocal z-series to increase vesicle resolution (see Methods). Our mouse experiments concerned 446 identification of precise environmental metabolite conditions that caused activation of the 
endocytosis and lysosome formation phenotype and the timing and cellular signalling activity involved. Collectively, our data firstly pinpoint the central role of isoleucine (ILE) deficiency, with more peripheral roles for other low BCAA and insulin levels in combination, as environmental factors which activate the histotrophic response in mouse TE. Second, we show these culture conditions promote the endocytic phenotype only from the time of blastocoel formation and involves recruitment of the TFEB transcription factor to enter the nucleus, mediated through mTORC1 downregulation. Lastly, we provided evidence of a similar environmental responsiveness in the human blastocyst, in this case through high maternal BMI as a measure of suboptimal nutrition, leading to increased lysosomal phenotype and nuclear localisation of TFEB.

The identification of a central role for extracellular ILE deficiency in activating the endocytosis phenotype is unexpected. The BCAAs share a similar structure of non-linear aliphatic side chains and catabolic pathway via $\alpha$-keto acid formation (Shimomura, et al. 2006, Zhang, et al. 2017). They also share several common amino acid transporters for exchange at the cell membrane (Bröer and Bröer 2017) and BCAA uptake via characterised transporter systems promoting mouse blastocyst development including mTORC1 signalling have been reported (Eckert, et al. 2012, Lamb and Leese 1994, Martin, et al. 2003, Van Winkle, et al. 2006). However, as signalling metabolites, LEU is recognised as having a more potent efficacy in stimulating mTORC1 than either ILE or VAL. BCAA activation of

467 mTORC1 is mediated through the Rag GTPases such that LEU binds to the inhibitory 468 regulator of Rags, Sestrin-2, to disrupt inhibition at higher potency than either ILE or VAL

469 (Melick and Jewell 2020, Wolfson, et al. 2016). Thus, in many examples of mTORC1 470 signalling of cellular metabolism and growth, LEU acts with greater potency than other 471 BCAAs (Tomiya, et al. 2007, Yoshizawa, et al. 2002) but in a minority of cases, ILE has 
472 been shown a more effective upstream activator, such as in mouse models for mammary cell

473 signalling and lactation performance (Liu, et al. 2017) and in anti-angiogenic signalling in the colon (Murata and Moriyama 2007). ILE supplementation of maternal drinking water in early mouse gestation has also been shown to affect fetal growth and birthweight (To, et al. 2020).

There is also evidence that metabolic effector pathways related to mTORC1 exhibit increased sensitivity to ILE than LEU as in glucose uptake and regulation in myotubes (Doi, et al. 2003) and hepatocytes (Xiao, et al. 2014), indicating the ratio of BCAA availability to have a critical influence on mTORC1-related metabolic activity (Duan, et al. 2017). Thus, in our endocytosis and lysosome model, the central role of ILE deficiency in its activation may reflect reduced mTORC1 signalling in combination with other metabolic factors such as glucose regulation, known to be affected in the LPD model both maternally (Eckert, et al. 2012, Kwong, et al. 2000) and in fetal offspring (Kwong, et al. 2007). involved increased levels of apically-localised megalin receptor and clathrin endocytic components in combination with increased cathepsin B lysosomal enzyme activity, visualised by MagicRed. The response activated only from the time of blastocyst formation. This likely reflects the requirement for epithelial maturation to be complete including tight junction sealing and meaningful transepithelial transport to commence which occurs at this stage

491 (Eckert and Fleming 2008, Fleming and Pickering 1985). The endocytic and lysosomal 492 response to L-ILE/N-INS culture also involved the re-localisation of TFEB from mostly cytoplasmic sites to the nucleus. TFEB is a member of the MiT-TFE family of helix-loophelix leucine-zipper transcription factors (Steingrímsson, et al. 2004). TFEB resides at the lysosome under conditions of nutrient availability in association with mTORC1 via Rab32 where it is phosphorylated and inactivated by the mTORC1 kinase; however, under 
497 conditions of nutrient deprivation, through BCAA sensing requiring the Rag-GTPase

498 regulator of mTORC1, TFEB is dephosphorylated and activated to translocate to nucleus to

499 promote gene expression and lysosome biogenesis (Drizyte-Miller, et al. 2020, Puertollano,

500 et al. 2018, Roczniak-Ferguson, et al. 2012, Settembre, et al. 2012). We also found TFEB

501 translocation to the nucleus was induced by rapamycin even in conditions of nutrient

502 availability (N-BCAA/N-INS) confirming nutrient sensing and response was regulated

503 through mTORC1.

504

505

Individual cell volume was found not to differ across treatments. However, cell

506 volume was positively correlated with endocytosis and lysosome vesicle number and

507 collective volume in the mouse study and was also an influencing cofactor identified in the

human study. Cell cycling and division induce stabilised partitioning of endo-lysosomal

509

organelles between daughter cells in relation to cell volume via cytoskeletal means (Carlton,

510 et al. 2020). Moreover, some cyclin-dependent kinases (CDKs) interact with lysosomes to

511 maintain their homeostasis (Ishii, et al. 2019, Nowosad, et al. 2020). The relationship found

512 between lysosome vesicle density and cell volume in mouse TE cells was evident in all

513 treatments and linked to cell cycle as shown by the approximate doubling of vesicle number

514 or collective volume in the morula ( 16-cell) versus unstimulated blastocyst ( $\sim 32$-cell).

515 Whilst ILE deficiency in the blastocyst raised endocytosis and lysosome vesicle density and

516 collective volume relative to controls, this occurred across the range of cell volume indicating

517 independence from cell volume. However, a subset of the L-ILE/N-INS data with high

518 vesicle number or collective volume also had large cell volume, indicating cell volume to be

519 a secondary factor associated with the lysosomal stimulation. Cell volume regulation in

520 mouse oocytes and early cleavage embryos is mediated through glycine and glutamine acting

521 as organic osmolytes and volume-regulated anion channels to export organic osmolytes 
where necessary (Baltz and Tartia 2010, Tscherner, et al. 2021). However, since these amino acids occur at similar levels in LPD and NPD blastocysts (Eckert, et al. 2012); were present at the same concentration in all the current culture media which were checked for osmolarity (250-260 mOsm); and acceptable and equivalent developmental potential occurred in all treatments, we believe the association not to be linked with perturbation. The additional increase in nuclear localisation of TFEB in response to L-ILE/N-INS was found not to be influenced by cell volume. This likely reflects the direct control of TFEB localisation by mTORC1 activity rather than cell cycle dynamics.

From our mouse studies we therefore conclude that poor nutrient availability is sensed by the TE once it is a functional epithelium at the blastocyst stage, in particular by ILE deficiency through mTORC1 leading to nuclear translocation of TFEB to coordinate increased endocytosis and lysosome biogenesis by transcriptional reprogramming to compensate by histotrophic nutrition of uterine fluid. The mouse TE endocytosis and lysosome phenotype therefore acts as a biomarker of adversely programmed embryos, whilst better protected for survival during gestation, having increased disease risk in later life. To explore the clinical relevance of these findings, the lysosome phenotype was assessed in human embryos donated for research.

For the human embryo analysis, culture in media with differing nutrient levels was not a feasible strategy since maternal heterogeneity in demographics would have confounded the analysis. Thus, normalised culture of embryos to form blastocysts was conducted and maternal BMI (high and normal groups) was used as a proxy for distinct nutritional environments. This preliminary study revealed increased collective volume of lysosome vesicles per TE cell in the high BMI group but with clear variation evident per embryo. 
547 Moreover, TFEB was distributed preferentially in the nucleus in blastocysts from high BMI

548 mothers, further indicating aspects of maternal metabolic status influence the embryo

549 phenotype. We also found that embryos from the same mother exhibited a similar lysosome

550 and TFEB staining pattern but other aspects of treatment, such as ICSI versus non-ICSI

551 fertilisation, or whether pregnancy resulted from that cycle, did not appear influential. This is

552 consistent with a similar metabolic profile occurring in embryos derived from ICSI and

553 conventional IVF (Leary and Sturmey 2020).

554

Whilst our evidence that maternal nutritional environment influences lysosome status and TFEB distribution in the human blastocyst is limited and requires a larger study for confirmation, it is consistent with deleterious effects and reduced fertility identified in oocytes and embryos from obese and high BMI mothers (Machtinger, et al. 2012, van der

Steeg, et al. 2008). Overweight mothers generate fewer and smaller oocytes that give rise to

560 blastocysts at a reduced rate and with fewer TE cells than those of normal BMI mothers

561 (Bartolacci, et al. 2019, Comstock, et al. 2015, Leary, et al. 2015). Blastocysts from overweight mothers also exhibit impaired glucose metabolism and increased triglyceride content (Leary, et al. 2015) reflecting increased accumulation of metabolites within follicular

564 fluid (Robker, et al. 2009). However, in contrast to our mouse study, the altered profile of

565 lysosomes and TFEB distribution is mediated not through deficiency in immediate culture

566 composition but from maternal metabolism. In this context, our human data is more similar to

567 our maternal LPD mouse model but with dietary condition being overnutrition rather than

568 protein restriction. High maternal BMI did not significantly alter the uterine fluid

569 composition of individual amino acids but generally increased their mean concentration

570 (Kermack, et al. 2015), in contrast to their reduction in the mouse LPD model (Eckert, et al.

571 2012). A maternal diet validated as less healthy than one with reduced fats and more 
572 vegetables and fruit also resulted in increased uterine fluid AAs with individual BCAAs

573 significantly increased (Kermack, et al. 2015). Interestingly, improved Mediterranean-style

574 diet has been shown to stimulate human embryo development and lead to increased

575 pregnancy over less healthy diets comprising higher fat intake (Braga, et al. 2015, Kermack,

576 et al. 2020, Vujkovic, et al. 2010). Thus, the increased lysosomal phenotype found in

577 blastocysts from the high BMI group may reflect more a stress response to over nutrition than

578 a deficiency in metabolites. Embryo screening for developmental potential in clinical ART is

579 predominantly restricted to morphological and morphokinetic approaches with limited scope

580 for embryo metabolic health (Ferrick, et al. 2020). Whilst the lysosomal phenotype of

581 adverse metabolic health revealed here depended upon invasive processing, advanced non-

582 invasive light microscopy technologies and imaging (eg, optical coherence microscopy;

583 (Karnowski, et al. 2017)) are emerging for visualisation of cytoplasmic organelles that may

584 lead to improved embryo selection.

585

586

In conclusion, we describe a critical early mechanism in mouse preimplantation

development to combat low maternal nutrient availability, activated especially by ILE

deficiency but also by depletion in other BCAAs and insulin in the blastocyst TE. This signal

leads to stimulation in endocytosis and lysosome biogenesis to increase compensatory

590 histotrophic nutrition mediated by reduced mTORC1 activity that promotes nuclear

591 translocation of the TFEB transcription factor. The mechanism associates with increased risk

592 of chronic disease in later life, hence is an early marker of adverse periconceptional programming. In a preliminary study, a similar capacity for increased lysosome biogenesis is

594 evident in human blastocysts activated in response to high maternal BMI and, if

595 substantiated, may provide a means to assess embryo metabolic potential in a clinical setting. 


\section{Declaration of interest}

598 The authors declare that there is no conflict of interest that could be perceived as prejudicing

599 the impartiality of the research reported.

600

$601 \quad$ Funding

602 This work was supported through the Rosetrees Trust (A798), the Biotechnology and

603 Biological Sciences Research Council (BBSRC) (BB/F007450/1) and the University of

604 Southampton to TPF.

605

606 Ethics

607 Mice were kept under UK Home Office Project license to T P F and local ethics approval in 608 accordance with the Animals (Scientific Procedures) Act of 1986 and associated Codes of

609 Practise. Human embryos were donated with consent from Wessex Fertility Clinic under a 610 Human Fertilisation and Embryology Authority research licence to T P F and J J E.

611

\section{Author contribution statement}

613 L C performed experiments, analysed data and wrote and edited the manuscript. J J E

614 provided embryo and statistical expertise, analysed data and edited the manuscript. D J, D S

615 C and N R S provided technical support and edited the manuscript. D A T provided cellular

616 expertise and edited the manuscript. S I and A P provided human embryo access and

617 expertise via HFEA research licence, and edited the manuscript. T P F conceived and

618 designed the study and wrote and edited the manuscript.

619

620 Acknowledgements 
We thank staff from the University of Southampton Biomedical Research Facility for animal facilities.

\section{References}

626

627

628

629

630

631

632

633

634

635

636

637

638

639

640

641

642

643

644

645

646

647

648

649

650

651

652

653

654

655

656

657

658

659

660

661

662

663

Assémat, E, S Vinot, F Gofflot, P Linsel-Nitschke, F Illien, F Châtelet, P Verroust, S Louvet-Vallée, F Rinninger, and R Kozyraki 2005 Expression and role of cubilin in the internalization of nutrients during the peri-implantation development of the rodent embryo. Biol Reprod 72 1079-1086.

Baltz, JM, and AP Tartia 2010 Cell volume regulation in oocytes and early embryos: connecting physiology to successful culture media. Hum Reprod Update 16 166-176.

Bartolacci, A, J Buratini, C Moutier, MC Guglielmo, PV Novara, F Brambillasca, MM Renzini, and M Dal Canto 2019 Maternal body mass index affects embryo morphokinetics: a time-lapse study. J Assist Reprod Genet 36 1109-1116.

Braga, DP, G Halpern, AS Setti, RC Figueira, A Iaconelli, Jr., and E Borges, Jr. 2015 The impact of food intake and social habits on embryo quality and the likelihood of blastocyst formation. Reprod Biomed Online 31 30-38.

Bröer, S, and A Bröer 2017 Amino acid homeostasis and signalling in mammalian cells and organisms. Biochem J 474 1935-1963.

Carlton, JG, H Jones, and US Eggert 2020 Membrane and organelle dynamics during cell division. Nat Rev Mol Cell Biol 21 151-166.

Coan, PM, OR Vaughan, J McCarthy, C Mactier, GJ Burton, M Constancia, and AL Fowden 2011 Dietary composition programmes placental phenotype in mice. $J$ Physiol 589 3659-3670.

Comstock, IA, S Kim, B Behr, and RB Lathi 2015 Increased body mass index negatively impacts blastocyst formation rate in normal responders undergoing in vitro fertilization. J Assist Reprod Genet 32 1299-1304.

Doi, M, I Yamaoka, T Fukunaga, and M Nakayama 2003 Isoleucine, a potent plasma glucose-lowering amino acid, stimulates glucose uptake in $\mathrm{C} 2 \mathrm{C} 12$ myotubes. Biochem Biophys Res Commun 312 1111-1117.

Drizyte-Miller, K, J Chen, H Cao, MB Schott, and MA McNiven 2020 The small GTPase Rab32 resides on lysosomes to regulate mTORC1 signaling. J Cell Sci 133.

Duan, Y, L Zeng, F Li, W Wang, Y Li, Q Guo, Y Ji, B Tan, and Y Yin 2017 Effect of branched-chain amino acid ratio on the proliferation, differentiation, and expression levels of key regulators involved in protein metabolism of myocytes. Nutrition 36 816.

Dunglison, GF, SD Jane, TF McCaul, JE Chad, TP Fleming, and PL Kaye 1995

Stimulation of endocytosis in mouse blastocysts by insulin: a quantitative morphological analysis. J Reprod Fertil 105 115-123.

Eckert, JJ, and TP Fleming 2008 Tight junction biogenesis during early development. Biochim Biophys Acta 1778 717-728.

Eckert, JJ, R Porter, AJ Watkins, E Burt, S Brooks, HJ Leese, PG Humpherson, IT Cameron, and TP Fleming 2012 Metabolic induction and early responses of mouse 
blastocyst developmental programming following maternal low protein diet affecting life-long health. PLoS One 7 e52791.

Ferrick, L, YSL Lee, and DK Gardner 2020 Metabolic activity of human blastocysts correlates with their morphokinetics, morphological grade, KIDScore and artificial intelligence ranking. Hum Reprod 35 2004-2016.

Feuer, S, and P Rinaudo 2016 From Embryos to Adults: A DOHaD Perspective on In Vitro Fertilization and Other Assisted Reproductive Technologies. Healthcare (Basel) 4.

Fleming, TP, and SJ Pickering 1985 Maturation and polarization of the endocytotic system in outside blastomeres during mouse preimplantation development. $J$ Embryol Exp Morphol 89 175-208.

Fleming, TP, AJ Watkins, MA Velazquez, JC Mathers, AM Prentice, J Stephenson, M Barker, R Saffery, CS Yajnik, JJ Eckert, et al 2018 Origins of lifetime health around the time of conception: causes and consequences. Lancet 391 1842-1852.

Gould, JM, PJ Smith, CJ Airey, EJ Mort, LE Airey, FDM Warricker, JE Pearson-Farr, EC Weston, PJW Gould, OG Semmence, et al 2018 Mouse maternal protein restriction during preimplantation alone permanently alters brain neuron proportion and adult short-term memory. Proc Natl Acad Sci US A.

Hanson, MA, and PD Gluckman 2014 Early developmental conditioning of later health and disease: physiology or pathophysiology? Physiol Rev 94 1027-1076.

Ishii, S, A Matsuura, and E Itakura 2019 Identification of a factor controlling lysosomal homeostasis using a novel lysosomal trafficking probe. Sci Rep 911635.

Jones, CB, EM Ott, JM Keener, M Curtiss, V Sandrin, and M Babst 2012 Regulation of membrane protein degradation by starvation-response pathways. Traffic 13 468-482.

Karnowski, K, A Ajduk, B Wieloch, S Tamborski, K Krawiec, M Wojtkowski, and M Szkulmowski 2017 Optical coherence microscopy as a novel, non-invasive method for the 4D live imaging of early mammalian embryos. Sci Rep 74165.

Kelleher, AM, FJ DeMayo, and TE Spencer 2019 Uterine Glands: Developmental Biology and Functional Roles in Pregnancy. Endocr Rev 40 1424-1445.

Kermack, AJ, S Finn-Sell, YC Cheong, N Brook, JJ Eckert, NS Macklon, and FD Houghton 2015 Amino acid composition of human uterine fluid: association with age, lifestyle and gynaecological pathology. Hum Reprod 30 917-924.

Kermack, AJ, P Lowen, SJ Wellstead, HL Fisk, M Montag, Y Cheong, C Osmond, FD Houghton, PC Calder, and NS Macklon 2020 Effect of a 6-week "Mediterranean" dietary intervention on in vitro human embryo development: the Preconception Dietary Supplements in Assisted Reproduction double-blinded randomized controlled trial. Fertil Steril 113 260-269.

Kim, J, G Song, G Wu, H Gao, GA Johnson, and FW Bazer 2013 Arginine, leucine, and glutamine stimulate proliferation of porcine trophectoderm cells through the MTORRPS6K-RPS6-EIF4EBP1 signal transduction pathway. Biol Reprod $\mathbf{8 8} 113$.

Kim, JY, RC Burghardt, G Wu, GA Johnson, TE Spencer, and FW Bazer 2011 Select nutrients in the ovine uterine lumen. VII. Effects of arginine, leucine, glutamine, and glucose on trophectoderm cell signaling, proliferation, and migration. Biol Reprod $\mathbf{8 4}$ 62-69.

Kleijkers, SH, E Mantikou, E Slappendel, D Consten, J van Echten-Arends, AM Wetzels, M van Wely, LJ Smits, AP van Montfoort, S Repping, et al 2016 Influence of embryo culture medium (G5 and HTF) on pregnancy and perinatal outcome after IVF: a multicenter RCT. Hum Reprod 31 2219-2230.

Kwong, WY, DJ Miller, AP Wilkins, MS Dear, JN Wright, C Osmond, J Zhang, and TP Fleming 2007 Maternal low protein diet restricted to the preimplantation period 
induces a gender-specific change on hepatic gene expression in rat fetuses. Mol Reprod Dev 74 48-56.

Kwong, WY, AE Wild, P Roberts, AC Willis, and TP Fleming 2000 Maternal undernutrition during the preimplantation period of rat development causes blastocyst abnormalities and programming of postnatal hypertension. Development 1274195 4202.

Lamb, VK, and HJ Leese 1994 Uptake of a mixture of amino acids by mouse blastocysts. $J$ Reprod Fertil 102 169-175.

Lanham, SA, SJ Smith, AJ Watkins, ES Lucas, N MacCaoilte, ROC Oreffo, TP Fleming, and JJ Eckert 2020 Periconception maternal low-protein diet adversely affects male mouse fetal bone growth and mineral density quality in late gestation. $J$ Dev Orig Health Dis 1-12.

Leary, C, HJ Leese, and RG Sturmey 2015 Human embryos from overweight and obese women display phenotypic and metabolic abnormalities. Hum Reprod 30 122-132.

Leary, C, and RG Sturmey 2020 Metabolic profile of in vitro derived human embryos is not affected by the mode of fertilization. Mol Hum Reprod 26 277-287.

Liu, GM, MD Hanigan, XY Lin, K Zhao, FG Jiang, RR White, Y Wang, ZY Hu, and ZH Wang 2017 Methionine, leucine, isoleucine, or threonine effects on mammary cell signaling and pup growth in lactating mice. J Dairy Sci 100 4038-4050.

Machtinger, R, CM Combelles, SA Missmer, KF Correia, JH Fox, and C Racowsky 2012 The association between severe obesity and characteristics of failed fertilized oocytes. Hum Reprod 27 3198-3207.

Martin, PM, AE Sutherland, and LJ Van Winkle 2003 Amino acid transport regulates blastocyst implantation. Biol Reprod 69 1101-1108.

Meads, TJ, and AE Wild 1993 Apical expression of an antigen common to rabbit yolk sac endoderm and kidney proximal tubule epithelium. J Reprod Immunol 23 247-264.

Melick, CH, and JL Jewell 2020 Regulation of mTORC1 by Upstream Stimuli. Genes (Basel) 11.

Murata, K, and M Moriyama 2007 Isoleucine, an essential amino acid, prevents liver metastases of colon cancer by antiangiogenesis. Cancer Res 67 3263-3268.

Nowosad, A, P Jeannot, C Callot, J Creff, RT Perchey, C Joffre, P Codogno, S Manenti, and A Besson 2020 p27 controls Ragulator and mTOR activity in amino aciddeprived cells to regulate the autophagy-lysosomal pathway and coordinate cell cycle and cell growth. Nat Cell Biol 22 1076-1090.

Puertollano, R, SM Ferguson, J Brugarolas, and A Ballabio 2018 The complex relationship between TFEB transcription factor phosphorylation and subcellular localization. $E M B O J 37$.

Robker, RL, LK Akison, BD Bennett, PN Thrupp, LR Chura, DL Russell, M Lane, and RJ Norman 2009 Obese women exhibit differences in ovarian metabolites, hormones, and gene expression compared with moderate-weight women. J Clin Endocrinol Metab 94 1533-1540.

Roczniak-Ferguson, A, CS Petit, F Froehlich, S Qian, J Ky, B Angarola, TC Walther, and SM Ferguson 2012 The transcription factor TFEB links mTORC1 signaling to transcriptional control of lysosome homeostasis. Sci Signal 5 ra42.

Sardiello, M, M Palmieri, A di Ronza, DL Medina, M Valenza, VA Gennarino, C Di Malta, F Donaudy, V Embrione, RS Polishchuk, et al 2009 A gene network regulating lysosomal biogenesis and function. Science 325 473-477.

Settembre, C, R Zoncu, DL Medina, F Vetrini, S Erdin, S Erdin, T Huynh, M Ferron, G Karsenty, MC Vellard, et al 2012 A lysosome-to-nucleus signalling mechanism senses and regulates the lysosome via mTOR and TFEB. EMBO J31 1095-1108. 
Shimomura, Y, Y Yamamoto, G Bajotto, J Sato, T Murakami, N Shimomura, H Kobayashi, and K Mawatari 2006 Nutraceutical effects of branched-chain amino acids on skeletal muscle. J Nutr $136529 \mathrm{~s}-532 \mathrm{~s}$.

Steingrímsson, E, NG Copeland, and NA Jenkins 2004 Melanocytes and the microphthalmia transcription factor network. Annu Rev Genet 38 365-411.

Sun, C, MA Velazquez, S Marfy-Smith, B Sheth, A Cox, DA Johnston, N Smyth, and TP Fleming 2014 Mouse early extra-embryonic lineages activate compensatory endocytosis in response to poor maternal nutrition. Development 141 1140-1150.

Sunde, A, D Brison, J Dumoulin, J Harper, K Lundin, MC Magli, E Van den Abbeel, and A Veiga 2016 Time to take human embryo culture seriously. Hum Reprod $\mathbf{3 1}$ 2174-2182.

Takahara, T, Y Amemiya, R Sugiyama, M Maki, and H Shibata 2020 Amino aciddependent control of mTORC1 signaling: a variety of regulatory modes. J Biomed Sci 2787.

To, CY, M Freeman, and LJ Van Winkle 2020 Consumption of a Branched-Chain Amino Acid (BCAA) during Days 2-10 of Pregnancy Causes Abnormal Fetal and Placental Growth: Implications for BCAA Supplementation in Humans. Int J Environ Res Public Health 17.

Tomiya, T, T Nishikawa, Y Inoue, N Ohtomo, H Ikeda, K Tejima, N Watanabe, Y Tanoue, M Omata, and K Fujiwara 2007 Leucine stimulates HGF production by hepatic stellate cells through mTOR pathway. Biochem Biophys Res Commun 358 176-180.

Tscherner, AK, AD Macaulay, CS Ortman, and JM Baltz 2021 Initiation of cell volume regulation and unique cell volume regulatory mechanisms in mammalian oocytes and embryos. J Cell Physiol.

Tsichlaki, E, and G FitzHarris 2016 Nucleus downscaling in mouse embryos is regulated by cooperative developmental and geometric programs. Sci Rep 628040.

van der Steeg, JW, P Steures, MJ Eijkemans, JD Habbema, PG Hompes, JM Burggraaff, GJ Oosterhuis, PM Bossuyt, F van der Veen, and BW Mol 2008 Obesity affects spontaneous pregnancy chances in subfertile, ovulatory women. Hum Reprod 23 324-328.

Van Winkle, LJ, JK Tesch, A Shah, and AL Campione 2006 System B0,+ amino acid transport regulates the penetration stage of blastocyst implantation with possible longterm developmental consequences through adulthood. Hum Reprod Update 12 145157.

Velazquez, MA, TP Fleming, and AJ Watkins 2019 Periconceptional environment and the developmental origins of disease. J Endocrinol 242 T33-t49.

Velazquez, MA, B Sheth, SJ Smith, JJ Eckert, C Osmond, and TP Fleming 2018 Insulin and branched-chain amino acid depletion during mouse preimplantation embryo culture programmes body weight gain and raised blood pressure during early postnatal life. Biochim Biophys Acta Mol Basis Dis 1864 590-600.

Vujkovic, M, JH de Vries, J Lindemans, NS Macklon, PJ van der Spek, EA Steegers, and RP Steegers-Theunissen 2010 The preconception Mediterranean dietary pattern in couples undergoing in vitro fertilization/intracytoplasmic sperm injection treatment increases the chance of pregnancy. Fertil Steril 94 2096-2101.

Watkins, AJ, ES Lucas, S Marfy-Smith, N Bates, SJ Kimber, and TP Fleming 2015 Maternal nutrition modifies trophoblast giant cell phenotype and fetal growth in mice. Reproduction 149 563-575. 
Watkins, AJ, ES Lucas, A Wilkins, FR Cagampang, and TP Fleming 2011 Maternal periconceptional and gestational low protein diet affects mouse offspring growth, cardiovascular and adipose phenotype at 1 year of age. PLoS One 6 e28745.

Watkins, AJ, D Platt, T Papenbrock, A Wilkins, JJ Eckert, WY Kwong, C Osmond, M Hanson, and TP Fleming 2007 Mouse embryo culture induces changes in postnatal phenotype including raised systolic blood pressure. Proc Natl Acad Sci U S A 104 5449-5454.

Watkins, AJ, E Ursell, R Panton, T Papenbrock, L Hollis, C Cunningham, A Wilkins, VH Perry, B Sheth, WY Kwong, et al 2008 Adaptive responses by mouse early embryos to maternal diet protect fetal growth but predispose to adult onset disease. Biol Reprod 78 299-306.

Wolfson, RL, L Chantranupong, RA Saxton, K Shen, SM Scaria, JR Cantor, and DM Sabatini 2016 Sestrin2 is a leucine sensor for the mTORC1 pathway. Science $35143-$ 48.

Xiao, F, J Yu, Y Guo, J Deng, K Li, Y Du, S Chen, J Zhu, H Sheng, and F Guo 2014 Effects of individual branched-chain amino acids deprivation on insulin sensitivity and glucose metabolism in mice. Metabolism 63 841-850.

Yoshizawa, F, S Hirayama, H Sekizawa, T Nagasawa, and K Sugahara 2002 Oral administration of leucine stimulates phosphorylation of 4E-bP1 and S6K 1 in skeletal muscle but not in liver of diabetic rats. J Nutr Sci Vitaminol (Tokyo) 48 59-64.

Zhang, S, X Zeng, M Ren, X Mao, and S Qiao 2017 Novel metabolic and physiological functions of branched chain amino acids: a review. J Anim Sci Biotechnol 810.

\section{Figure legends}

Figure 1. Endocytosis and lysosomes are increased in mouse blastocyst TE following culture from 2-cell stage in medium with depleted Isoleucine alone and normal Insulin (L-ILE/N-

INS). A. Blastocysts z-series after BSA-BODIPY (green) and LysoTracker (red) endocytosis and lysosome assay and nuclei (DAPI, blue) staining following culture in different BCAAs concentrations - either at the level found in NPD uterine fluid (N-BCAA), or with one BCAA individually reduced by 50\% compared to N-BCAA (L-VAL/N-INS, L-LEU/N-INS and LILE/N-INS) or with normal concentration of BCAAs but 50\% decreased insulin (L-INS).

Scale bar $=20 \mu \mathrm{m}$. B-E. LysoTracker and BSA-BODIPY vesicle number and collective volume $\left(\mu \mathrm{m}^{3}\right)$ per TE cell. Endocytosis and lysosome number are stimulated in the L-ILE/NINS group but not significantly in the other treatment groups. Data were analysed by 1-way ANOVA and shown as dot plots with box and whisker markers to identify the median and 
interquartile range and $\min / \max$ points. ${ }^{*} \mathrm{P}<0.05$; for other $\mathrm{P}$ values, see text; $17-24$ embryos per group (30-55 cells per group) from 13 mothers (in 10 replicates). Abbreviations: NBCAA/N-INS Normal BCAA, normal insulin; L-VAL/N-INS low valine, normal insulin; LLEU/N-INS low leucine, normal insulin; L-ILE/N-INS low isoleucine, normal insulin; NBCAA/L-INS normal BCAA, low insulin.

852

Figure 2. Cathepsin B (MagicRed) activity and megalin (red) and clathrin (green) immunostaining are increased in mouse blastocyst TE following culture from 2-cell stage in media with depleted Isoleucine (L-ILE/N-INS) A. Blastocysts after MagicRed (red) and LysoTracker (green) assay and nuclei (DAPI, blue) staining following culture in either the BCAA level found in NPD uterine fluid (N-BCAA), or with reduced (50\%) Isoleucine (LILE). B,C. Cathepsin B vesicle number and collective volume per TE cell is increased in the L-ILE/N-INS group. 21-26 embryos per group from 7 mothers (in 4 replicates).

D. NBCAA/N-INS and E. L-ILE/N-INS blastocysts stained for megalin (red), clathrin (green) and nuclei (DAPI, blue). High magnification of TE layer shown below; markers are localised to apical TE and cytoplasm. F. Megalin and G. Clathrin staining intensity (voxels) is increased in L-ILE/N-INS blastocyst TE cells. 10-18 embryos per group from 6 mothers in 4 replicates.

864 Scale bar $=20 \mu \mathrm{m}$. Data were analysed by t-test $(\mathbf{B}, \mathbf{C})$ and Mann-Whitney $(\mathbf{F}, \mathbf{G})$ and shown

865 as dot plots with box and whisker markers to identify the median and interquartile range and $\min / \max$ points. ${ }^{*} \mathrm{P}<0.05,{ }^{*} \mathrm{P}<0.01$; for other $\mathrm{P}$ values, see text. Abbreviations: $\mathrm{N}$ BCAA/N-INS Normal BCAA, normal insulin; L-ILE/N-INS low isoleucine, normal insulin.

869 Figure 3. Endocytosis is increased in mouse blastocyst TE following culture from 2-cell 870 stage in media with L-ILE/N-INS and L-ILE/L-LEU/L-INS. Culture in L-BCAA/L-INS increased endocytosis to a trend level. A. Representative blastocysts after BSA-BODIPY 
872 (green) and LysoTracker (red) endocytosis assay and nuclei (DAPI, blue) staining following

873 culture in different combinations of BCAAs and insulin concentrations - BCAAs were either

874 at the level found in NPD uterine fluid (N-BCAA), with one BCAA individually reduced by

$87550 \%$ (L-ILE or L-LEU), with two BCAA reduced by 50\% (L-ILE and L-LEU) or with all

876 BCAA reduced by $50 \%$ (L-BCAA) either with normal insulin (N-INS) or with $50 \%$

877 decreased insulin (L-INS). Scale bar $=20 \mu \mathrm{m}$. B,C. LysoTracker and D,E. BSA-BODIPY

878 vesicles number and collective volume $\left(\mu \mathrm{m}^{3}\right)$ per TE cell. Endocytosis is stimulated in the L-

879 ILE/N-INS and L-ILE/L-LEU groups but not significantly in the other depleted media.

880 Embryos cultured in L-BCAA/L-INS had a trend to increase endocytosis. Data were analysed

881 by Kruskal-Wallis (B-D) and 1-way ANOVA (E) and shown as dot plots with box and

882 whisker markers to identify the median and interquartile range and $\min /$ max points. ${ }^{*} \mathrm{P}<0.05$,

$883 * * \mathrm{P}<0.01$; for other $\mathrm{P}$ values, see text; 13-19 embryos per group (23-38 cells per group) from

88414 mothers (in 8 replicates). Abbreviations: N-BCAA/N-INS Normal BCAA, normal insulin;

885 L-ILE/N-INS low isoleucine, normal insulin; L-BCAA/L-INS low BCAA, low insulin; L-

886 ILE/L-INS low isoleucine, low insulin; L-LEU/L-INS low leucine, low insulin; L-ILE/L-

887 LEU/L-INS low isoleucine, low leucine, low insulin.

888

889 Figure 4. Lysosomes are not increased in mouse morulae outer cells following culture from

890 2-cell stage in media with depleted Isoleucine alone and normal insulin (L-ILE/N-INS). A.

891 Morulae after LysoTracker (LysTr, red) lysosome assay following culture in (N-BCAA/N-

892 INS; L-ILE/N-INS). Blastocysts used as control (embryos from same experiment left in

893 culture for $12 \mathrm{~h}$ more). Scale bar $=20 \mu \mathrm{m}$. B,C. Morula LysoTracker vesicle number and

894 collective volume $\left(\mu \mathrm{m}^{3}\right)$ per outer cell. D,E. Blastocyst LysoTracker vesicle number and

895 collective volume $\left(\mu \mathrm{m}^{3}\right)$ per TE cell. Data were analysed by Mann-Whitney and shown as dot

896 plots with box and whisker markers to identify the median and interquartile range and 
$897 \mathrm{~min} / \max$ points. ${ }^{* *} \mathrm{P}<0.01$; for other $\mathrm{P}$ values, see text. 17-18 embryos per group (24-26

898 cells per group) from 5 mothers (in 4 replicates). Abbreviations: N-BCAA/N-INS Normal

899 BCAA, normal insulin; L-ILE/N-INS low isoleucine, normal insulin.

900

901 Figure 5. TFEB staining in N-BCAA/N-INS mouse blastocysts is more cytoplasmic while in

902 L-ILE/N-INS it is more nuclear. A. TFEB staining in blastocysts following culture from 2-

903 cell stage. Scale bar $=20 \mu \mathrm{m}$. B,C. Percentage (\%) TFEB localised in the cytoplasm $(\mathbf{C})$ and

904 in the nucleus (D). E. Pearson's correlation for green and blue channel. L-ILE/N-INS TE

905 cells have increased proportion of the TFEB (green channel) co-labelled with the nucleus

906 (DAPI - blue channel). Data were analysed by t-test $(\mathbf{B}, \mathbf{C})$ and Mann-Whitney (D) and

907 shown as dot plots with box and whisker markers to identify the median and interquartile

908 range and $\mathrm{min} / \mathrm{max}$ points. $* \mathrm{P}<0.05$; for other $\mathrm{P}$ values, see text; 7-8 embryos per group (9-

90910 cells per group) from 4 mothers (in 4 replicates). Abbreviations: N-BCAA/N-INS Normal

910 BCAA, normal insulin; L-ILE/N-INS low isoleucine, normal insulin.

911

912 Figure 6. A. 2-cell mouse embryos cultured until blastocyst stage in N-BCAA/N-INS

913 medium supplemented with rapamycin at different concentrations $-0,1$ or $20 \mu \mathrm{M}$ before

914 immunolabelling for TFEB (green) and DAPI (blue). Scale bar $=20 \mu \mathrm{m}$. B. Percentage (\%)

915 TFEB localisation in nuclei rather than cytoplasm increased with Rapamycin treatment. C.

916 Pearson correlation showing increased co-localisation between TFEB and DAPI (nuclear

917 staining) as rapamycin concentration increases. Data were analysed by Kruskal-Wallis and

918 shown as dot plots with box and whisker markers to identify the median and interquartile

919 range and $\min / \max$ points. $* \mathrm{P}<0.05$, $* * * \mathrm{P}<0.001$; for other $\mathrm{P}$ values, see text. $6-13$ embryos

920 per group from 5 mothers (in 4 replicates). Abbreviations: N-BCAA/N-INS Normal BCAA,

921 normal insulin. 
923 Figure 7. A. 2-cell stage mouse embryos cultured to blastocyst stage in N-BCAA/N-INS

924 medium supplemented with rapamycin at different concentrations, $0,100 \mathrm{~nm}, 1$ or $20 \mu \mathrm{M}$,

925 before LysoTracker analysis (LysTr, red) and DAPI (blue). Scale bar $=20 \mu \mathrm{m}$. B.

926 LysoTracker collective volume/cell is not significantly changed by rapamycin although

927 slightly increased at $20 \mu \mathrm{M}$. Data were analysed by Kruskal-Wallis test and shown as dot

928 plots with box and whisker markers to identify the median and interquartile range and

$929 \mathrm{~min} / \mathrm{max}$ points. For select $\mathrm{P}$ values, see text; 6-13 embryos per group from 5 mothers (in 4

930 replicates). Abbreviations: N-BCAA/N-INS Normal BCAA, normal insulin.

931

932 Figure 8. Mouse blastocyst cell volume and correlation analyses between cell volume and endocytosis/lysosome factors for N-BCAA/N-INS (blue) and L-ILE/N-INS treatments (red).

A. Cell volume is not significantly changed between treatment groups assayed by LysoTracker and BSA-BODIPY. B,C. Cell volume and LysoTracker number and collective volume, respectively, are positively and significantly correlated in N-BCAA/N-INS and LILE/N-INS with LysoTracker data increased in the L-ILE/N-INS group. D,E. Cell volume and BSA-BODIPY number and collective volume, respectively, are positively correlated in N-BCAA/N-INS and L-ILE/N-INS with BSA-BODIPY data increased in the L-ILE/N-INS group. F. Cell volume is not significantly changed between N-BCAA/N-INS and L-ILE/NINS groups assayed for TFEB. G. No correlation was found between cell volume and the percentage of TFEB in the nucleus in both groups. Data were analysed by Kruskal-Wallis

943 (A), correlation (B-E,G) or $\mathrm{t}$ test $(\mathbf{F})$ and shown as dot plots with box and whisker markers to 944 identify the median and interquartile range and $\min / \max$ points $(\mathbf{A}, \mathbf{F})$ or dot plot correlation analysis (B-E,G). 7-8 embryos per group (9-10 cells per group) from 4 mothers (in 4

946 replicates). Abbreviations: N-BCAA/N-INS Normal BCAA, normal insulin; L-VAL/N-INS 
947 low valine, normal insulin; L-LEU/N-INS low leucine, normal insulin; L-ILE/N-INS low

948 isoleucine, normal insulin; N-BCAA/L-INS normal BCAA, low insulin.

949

950 Figure 9. LysoTracker collective volume per cell in human blastocyst TE from normal and

951 high BMI patients. A. Top TE cells labelled with LysoTracker (LysTr, red) and DAPI; scale

952 bar $=40 \mu \mathrm{m}$; single TE cell analysed in VOLOCITY software; scale bar $=5 \mu \mathrm{m}$. B.

953 LysoTracker volume per TE cell in blastocysts is higher for high BMI than normal BMI

954 patients. Each point is a single blastocyst and patient ID number is given alongside as in

955 Table 2. Mother ID was a cofactor in statistical analysis to account for possible similarity of

956 embryos from the same mother. E,F. Cell number and cell volume, respectively, are not

957 different between blastocysts from normal and high BMI patients. However, cell volume and

958 number were significantly influencing cofactors. Data were analysed by multilevel random

959 effects regression and shown as dot plots with mean and SEM. $* \mathrm{P}<0.05$; for other $\mathrm{P}$ values,

960 see text; 13-15 embryos per group from 7 mothers per group.

961

962 Figure 10. TE cells from human blastocysts stained with TFEB (green) and DAPI (blue)

963 from normal and high BMI patients. A. TFEB is mainly located in the cytoplasm in embryos

964 from normal BMI mothers while more TFEB staining co-localizes with the nucleus in high

965 BMI mothers. Scale bar $=5 \mu \mathrm{m}$. B. Pearson correlation showing increased mean co-

966 localisation between TFEB and DAPI (nuclear staining) in high versus normal BMI patients

967 but not to significance. Numbers represent Patient ID from Table 2. C,D. LysoTracker

968 collective volume and TFEB nuclear co-localisation (Pearson correlation), respectively, are

969 not different when comparing embryos derived from ICSI or not. Normal (blue) and high

970 (red) BMI patients are shown. Numbers represent Patient ID from Table 2. Data were 
analysed by multilevel random effects regression and shown as dot plots with mean and

SEM. For select P values, see text;13-14 embryos per group from 7 mothers per group.

973

974 Supplemental Figure 1. Mouse blastocyst LysoTracker (A, C) and BSA-BODIPY (B, D)

975 individual vesicle volumes and their distance from nucleus were not altered in response to

976 different culture treatments. Embryos were cultured from 2-cell stage and analysed at

977 blastocyst stage. A, B. Mean vesicle volume did not differ between groups. C, D. Mean

978 vesicle distance to nucleus did not differ between groups. E. Percentage (\%) of embryos

979 developing to blastocysts following culture from 2-cell stage after $48 \mathrm{~h}$ did not differ between

980 treatments. F. Cell number in blastocysts did not differ between treatments. Data were

981 analysed by Kruskal-Wallis (A,C,E) or 1-way ANOVA (B,D,F) and shown as dot plots with

982 box and whisker markers to identify the median and interquartile range and $\mathrm{min} / \mathrm{max}$ points.

983 17-24 embryos per group (30-55 cells per group) from 13 mothers (in 10 replicates).

984

985 Supplemental Figure 2. Mouse blastocyst LysoTracker (A,C) and BSA-BODIPY (B,D)

986 individual vesicle volumes and their distance from nucleus were not altered in response to

987 different culture treatments. Embryos were cultured from 2-cell stage and analysed at

988 blastocyst stage. A,B. Mean vesicle volume did not differ between groups. C,D. Mean vesicle

989 distance to nucleus did not differ between groups. Data were analysed by 1-way ANOVA and

990 shown as dot plots with box and whisker markers to identify the median and interquartile

991 range and $\mathrm{min} / \mathrm{max}$ points. 13-19 embryos per group (23-38 cells per group) from 14 mothers

992 (in 8 replicates).

993 
Table 1. Amino acid concentrations present in vivo in the NPD mother uterine fluid at E3.5 (Eckert et al., 2012) and used in the N-BCA medium.

\begin{tabular}{|cc|}
\hline AMINO ACIDS & $\begin{array}{c}\text { Concentration } \\
(\mathrm{mM})\end{array}$ \\
\hline Alanine & 3.8 \\
Arginine & 0.16 \\
Asparagine & 0.14 \\
Aspartic acid & 1.83 \\
Glutamic acid & 4.72 \\
Glutamine & 1.41 \\
Glycine & 2.68 \\
Histidine & 0.14 \\
Isoleucine & 0.21 \\
Leucine & 0.32 \\
Lysine & 0.5 \\
Methionine & 0.18 \\
Phenylalanine & 0.14 \\
Serine & 0.97 \\
Taurine & 14.74 \\
Threonine & 0.72 \\
Tryptophan & 0.06 \\
Tyrosine & 0.18 \\
Valine & 0.45 \\
\hline
\end{tabular}


Table 2. Patient and human embryo criteria, A Normal BMI; B High BMI. ICSI, intracytoplasmic sperm injection. Condition: PCOS, polycystic ovary syndrome; Low S+O, low sperm and ovarian reserve; Male Inf, male infertility; Unexpl, unexplained; Tubal, tubal blockage.

\begin{tabular}{|c|c|c|c|c|c|c|c|c|}
\hline \multirow{2}{*}{ A } & \multicolumn{8}{|c|}{ Normal BMI } \\
\hline & Patient 1 & Patient 2 & Patient 3 & Patient 4 & Patient 5 & Patient 6 & Patient 7 & Mean \\
\hline BMI & 18.97 & 21.72 & 22.94 & 21.91 & 23.1 & 22.48 & 22.14 & 21.90 \\
\hline Age & 31 & 35 & 27 & 38 & 33 & 35 & 35 & 33.75 \\
\hline Condition & PCOS & Low $\mathrm{S}+\mathrm{O}$ & Male Inf & Unexpl & Male Inf & Tubal & Unexpl & \\
\hline $\begin{array}{l}\text { Number } \\
\text { embryos }\end{array}$ & 8 & 3 & 10 & 7 & 4 & 6 & 4 & Total 42 \\
\hline Stage stored & D3 & $\begin{array}{l}\text { D3 (2) } \\
\text { D5 (1) }\end{array}$ & D3 & D5 & D3 & $\begin{array}{l}\text { D3 (3) } \\
\text { D5 (3) }\end{array}$ & D5 & \\
\hline ICSI & $\mathrm{NO}$ & YES & YES & $\mathrm{NO}$ & YES & NO & NO & $5 \mathrm{NO} / 3 \mathrm{YES}$ \\
\hline $\begin{array}{l}\text { Pregnancy } \\
\text { that cycle }\end{array}$ & YES & NO & NO & YES & YES & NO & YES & $3 \mathrm{NO} / 5 \mathrm{YES}$ \\
\hline $\begin{array}{c}\text { Blastocysts } \\
\text { formed }\end{array}$ & 3 & 1 & 3 & 3 & 1 & 2 & 2 & Total $15(36 \%)$ \\
\hline
\end{tabular}

\begin{tabular}{|c|c|c|c|c|c|c|c|c|}
\hline \multirow{2}{*}{ B } & \multicolumn{8}{|c|}{ High BMI } \\
\hline & Patient 8 & Patient 9 & Patient 10 & Patient 11 & Patient 12 & Patient 13 & Patient 14 & Mean \\
\hline BMI & 32.39 & 31.25 & 31.16 & 30.12 & 27.25 & 27.15 & 29.76 & 29.54 \\
\hline Age & 36 & 35 & 34 & 35 & 34 & 36 & 35 & 34.7 \\
\hline Condition & $\begin{array}{l}\text { PCOS }+ \\
\text { Male Inf } \\
\end{array}$ & Unexpl & $\begin{array}{c}\text { Tubal + } \\
\text { Male Inf }\end{array}$ & Unexpl & PCOS & Tubal & PCOS & \\
\hline $\begin{array}{l}\text { Number } \\
\text { embryos }\end{array}$ & 4 & 4 & 4 & 7 & 9 & 8 & 3 & Total 39 \\
\hline Stage stored & $\begin{array}{l}\text { D4 (2) } \\
\text { D5 (2) }\end{array}$ & D3 & D3 & D3 & $\begin{array}{l}\text { D3 (7) } \\
\text { D5(2) }\end{array}$ & $\begin{array}{l}\text { D3 (6) } \\
\text { D5 (2) }\end{array}$ & D3 & \\
\hline ICSI & YES & YES & YES & YES & NO & $\mathrm{NO}$ & YES & $4 \mathrm{NO} / 5 \mathrm{YES}$ \\
\hline $\begin{array}{l}\text { Pregnancy } \\
\text { that cycle }\end{array}$ & YES & YES & YES & NO & YES & YES & NO & 7 YES / 2 NO \\
\hline $\begin{array}{c}\text { Blastocysts } \\
\text { formed }\end{array}$ & 1 & 1 & 2 & 3 & 4 & 3 & 2 & Total $16(41 \%)$ \\
\hline
\end{tabular}


Supplementary Table 1. VOLOCITY protocols used for quantitative image analysis.

\section{(a) LysoTracker or MagicRed ${ }^{\mathrm{TM}}$ and BSA-BODIPY analysis}

1. Crop cell

2. Find object (based on channel) - red channel for LysoTracker Red or MagicRed ${ }^{\mathrm{TM}}$; green channel for BSA-BODIPY; blue channel (DAPI - nucleus)

3. Remove noise from objects (fine filter)

4. Separate touching objects (by $2 \mu \mathrm{m}$ )

5. Exclude objects by size: chosen based on the literature, according to Tsichlaki and

Fitzharris, (2016) the blastocyst nuclei size is $690 \pm 20 \mu \mathrm{m}^{3}$ while the lysosome size range is between $0.1-1.2 \mu \mathrm{m}$ diameter (Kuehnel, 2003)

5.1. vesicles $<0.01 \mu \mathrm{m}^{3}$ and $>20 \mu \mathrm{m}^{3}$

5.2. nuclei $<150 \mu \mathrm{m}^{3}$

6. Nuclei - fill holes in objects

7. Objects of interest were selected by automatic threshold using an offset of $50 \%$.

8. Measure distance from vesicle centre to nucleus edge (TE).

9. Object data were exported to Excel for analysis.

\section{(b) TFEB analysis}

1. Crop cell

2. Find object (based on channel) - blue channel (DAPI - nucleus) and green channel for TFEB

3. Remove noise from objects (fine filter)

4. Separate touching objects (by $2 \mu \mathrm{m}$ )

5. Exclude objects by size: chosen based on the literature, according to Tsichlaki and

Fitzharris, (2016) the blastocyst nuclei size is $690 \pm 20 \mu \mathrm{m}^{3}$

5.1. nuclei $<150 \mu \mathrm{m}^{3} \mathrm{Nuclei}-$ fill holes in objects

6. Objects of interest were selected by automatic threshold using an offset of $50 \%$.

7. Define cytoplasm: subtract nucleus from ROIs (cell)

8. Find TFEB in nucleus: intersect nucleus with TFEB

8.1. Remove noise from objects (fine filter)

8.2. Separate touching objects (by $2 \mu \mathrm{m}$ )

9. Find TFEB in cytoplasm: intersect cytoplasm with TFEB

9.1. Remove noise from objects (fine filter) 
9.2. Separate touching objects (by $2 \mu \mathrm{m}$ )

10. Colocalization: automatic threshold (Costes) - Costes Pearson correlation 11. Object data were exported to Excel for analysis. 
Figure 1

A

N-BCAA/N-INS

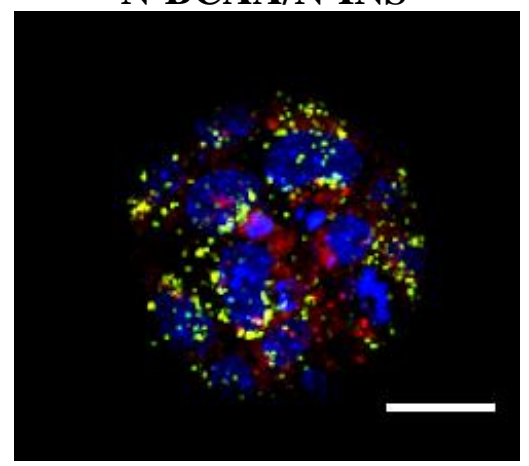

L-VAL/N-INS

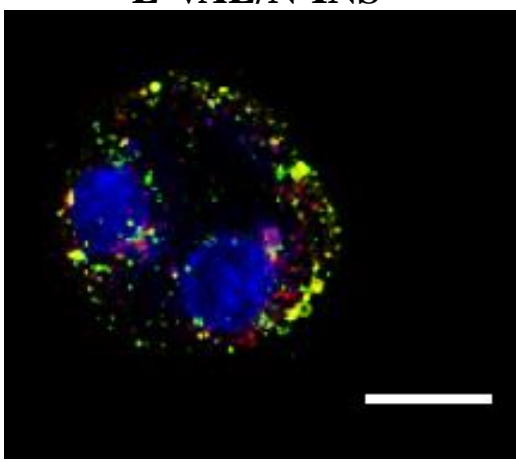

L-LEU/N-INS

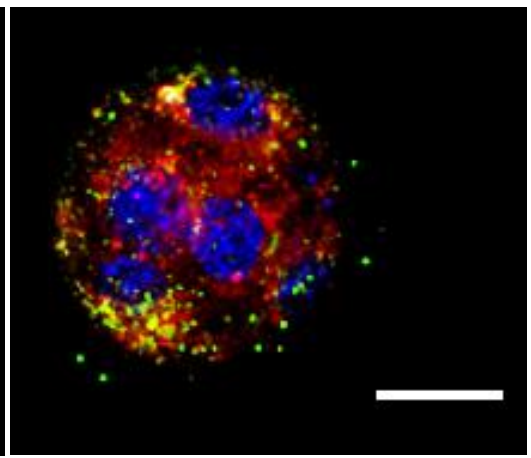

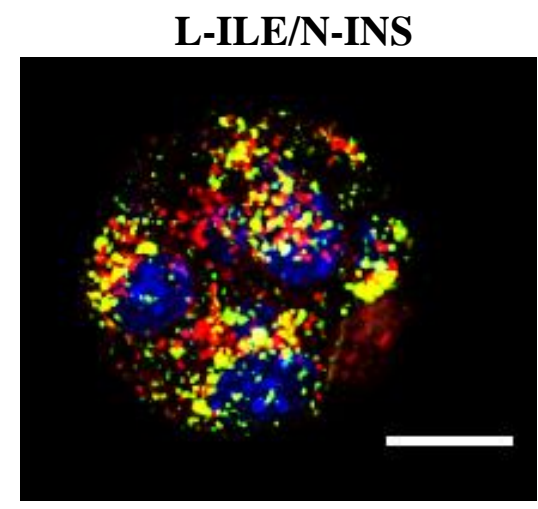

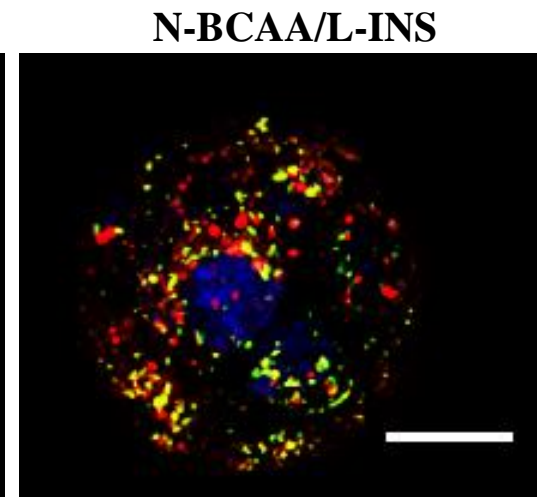

B

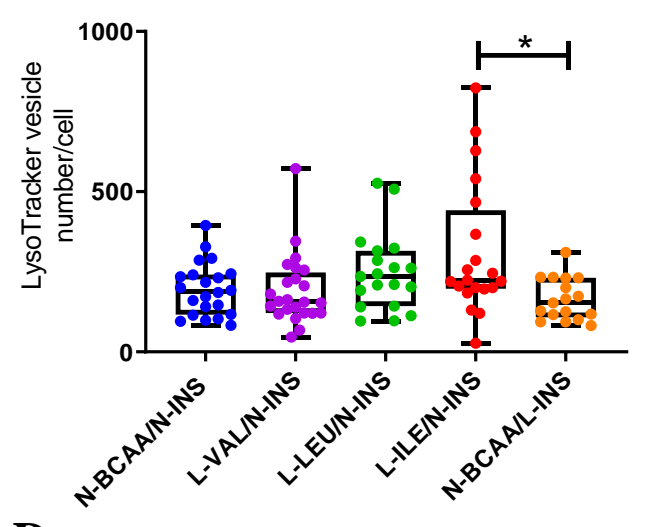

D

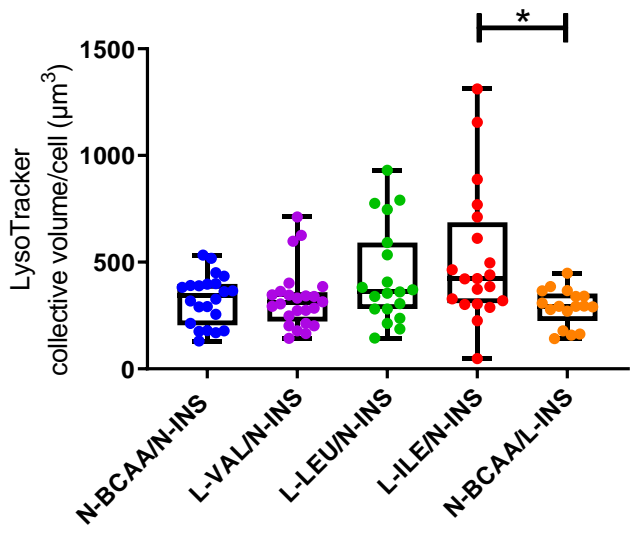

C

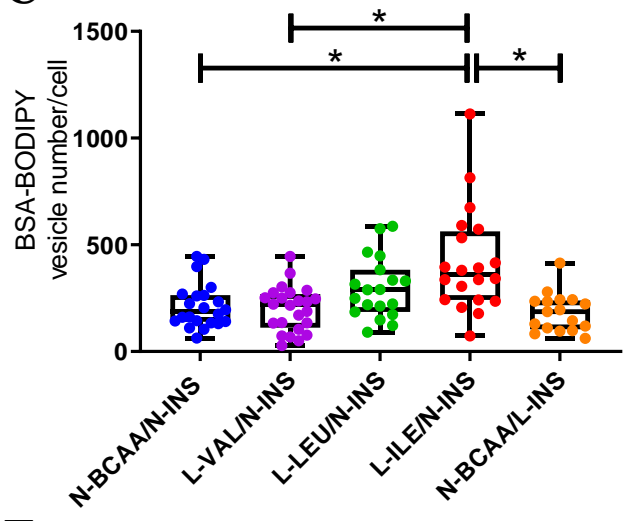

E

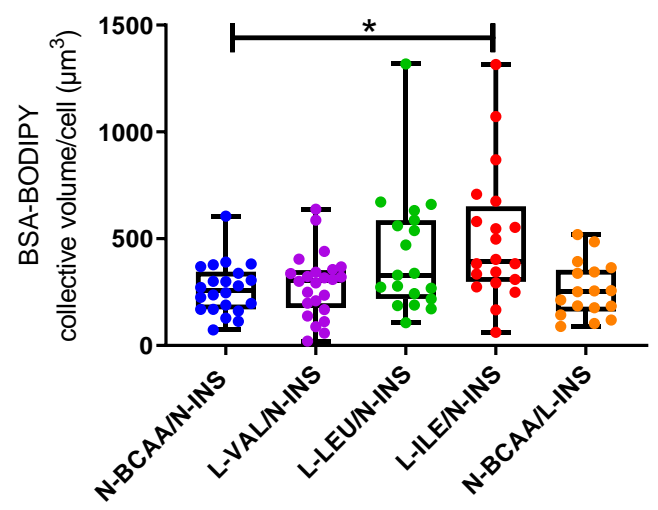


Figure 2

A

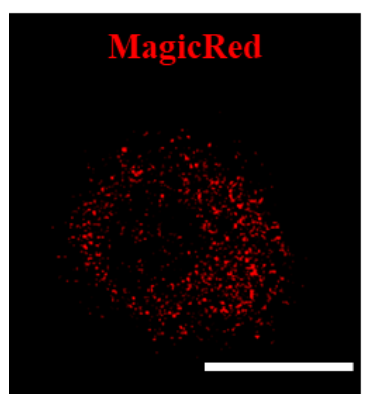

N-BCAA/N-INS
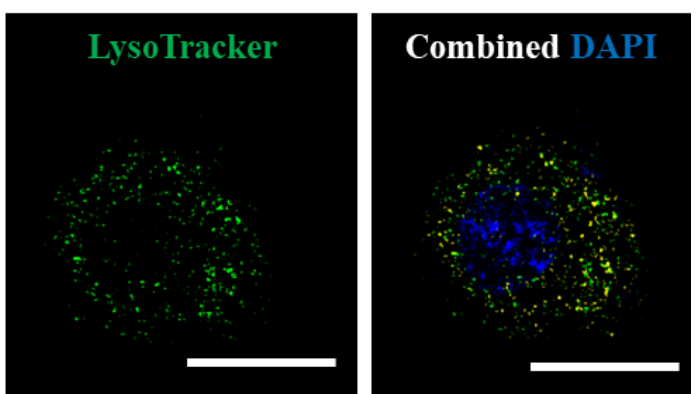

\section{L-ILE/N-INS}
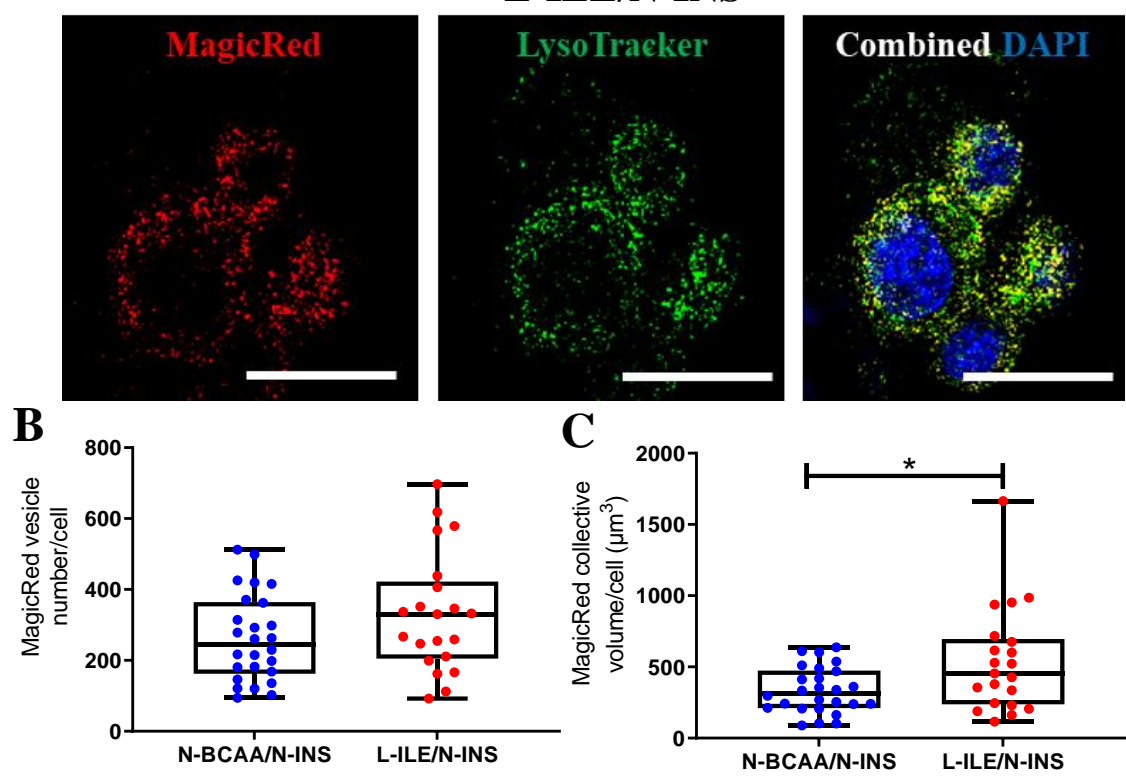

D

N-BCAA/N-INS
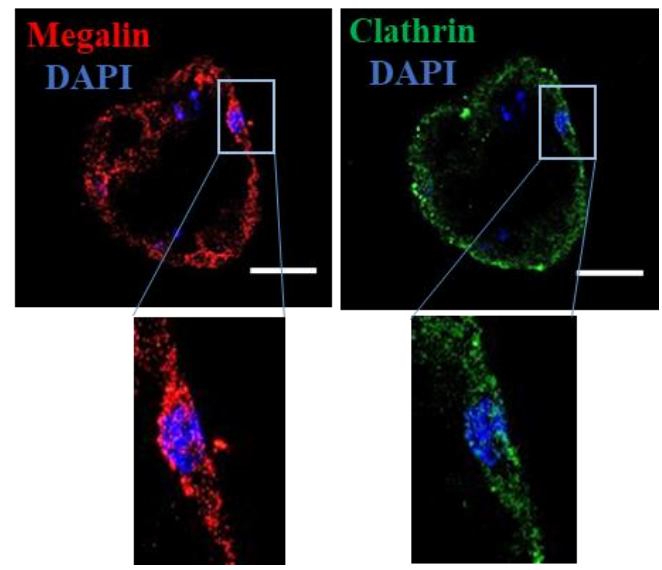

E

L-ILE/N-INS
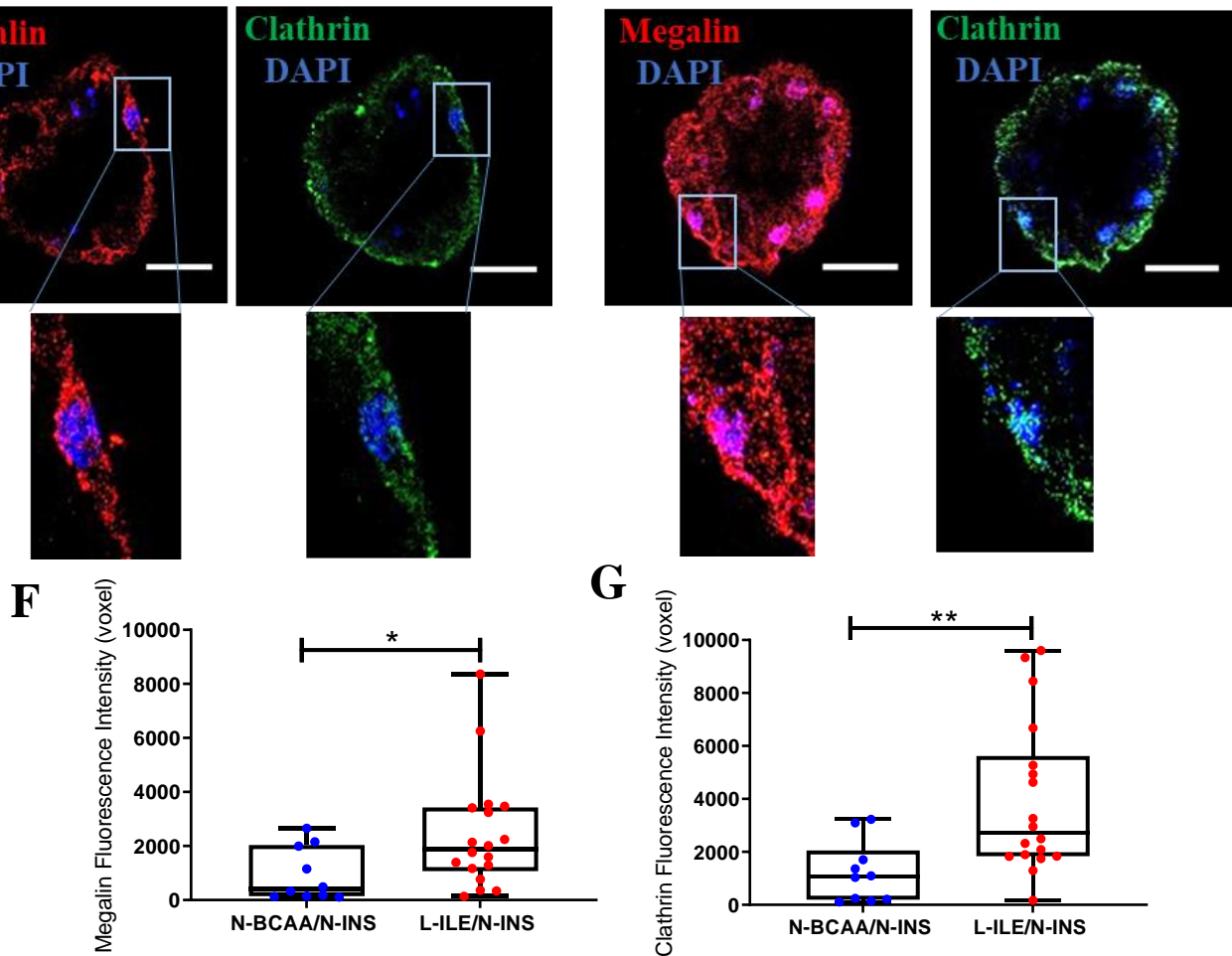


\section{Figure 3}

A

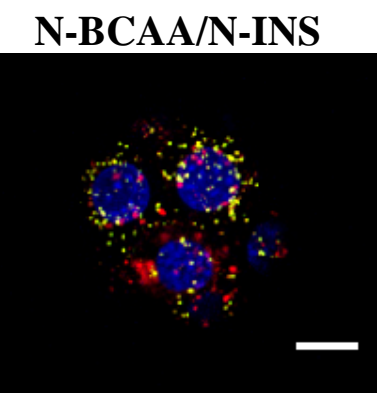

L-ILE/N-INS

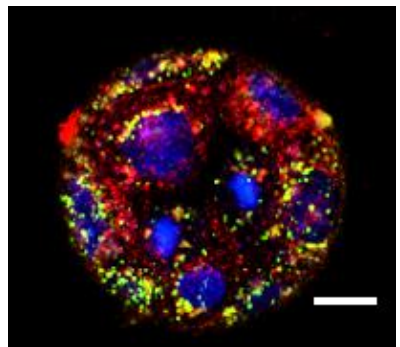

L-BCAA/L-INS

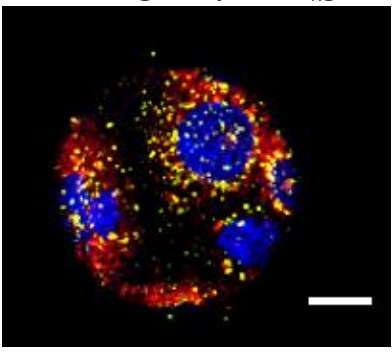

\section{L-ILE/L-INS}

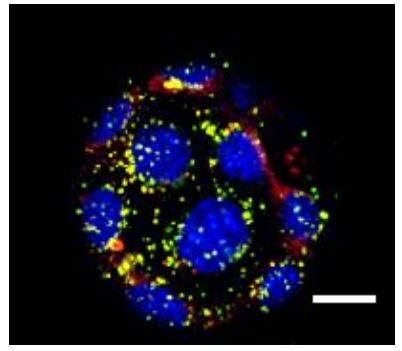

L-LEU/L-INS

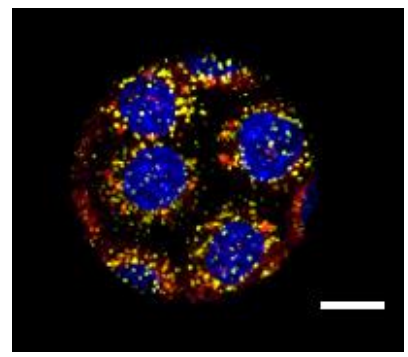

L-ILE/L-LEU/L-INS

B

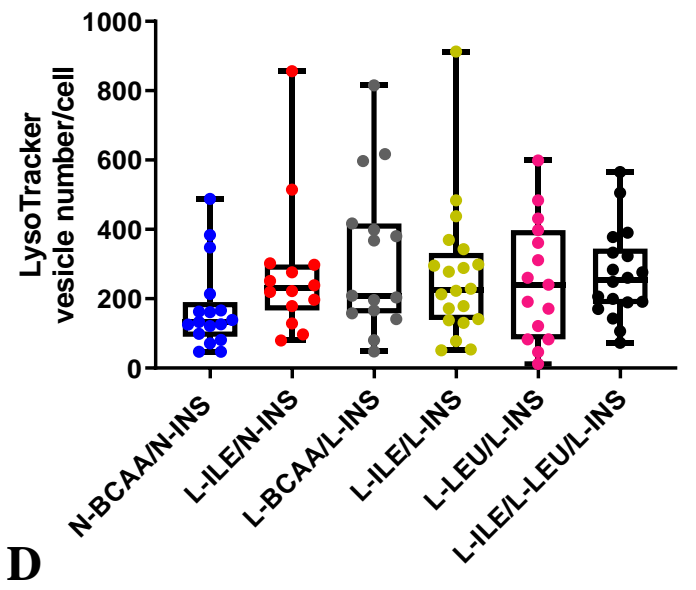

C

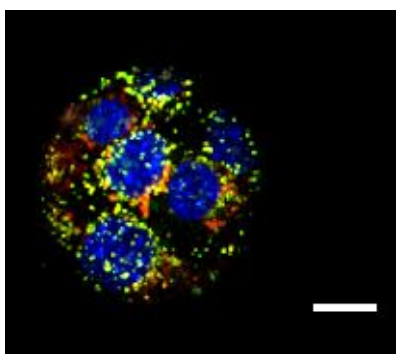

B

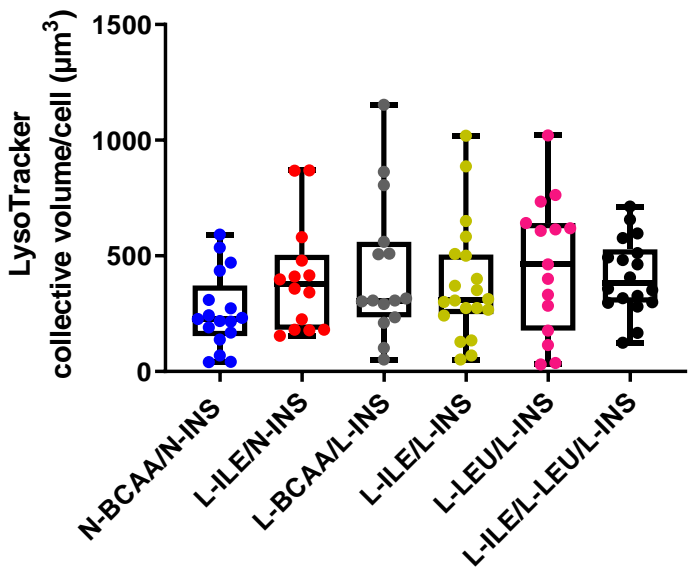

E
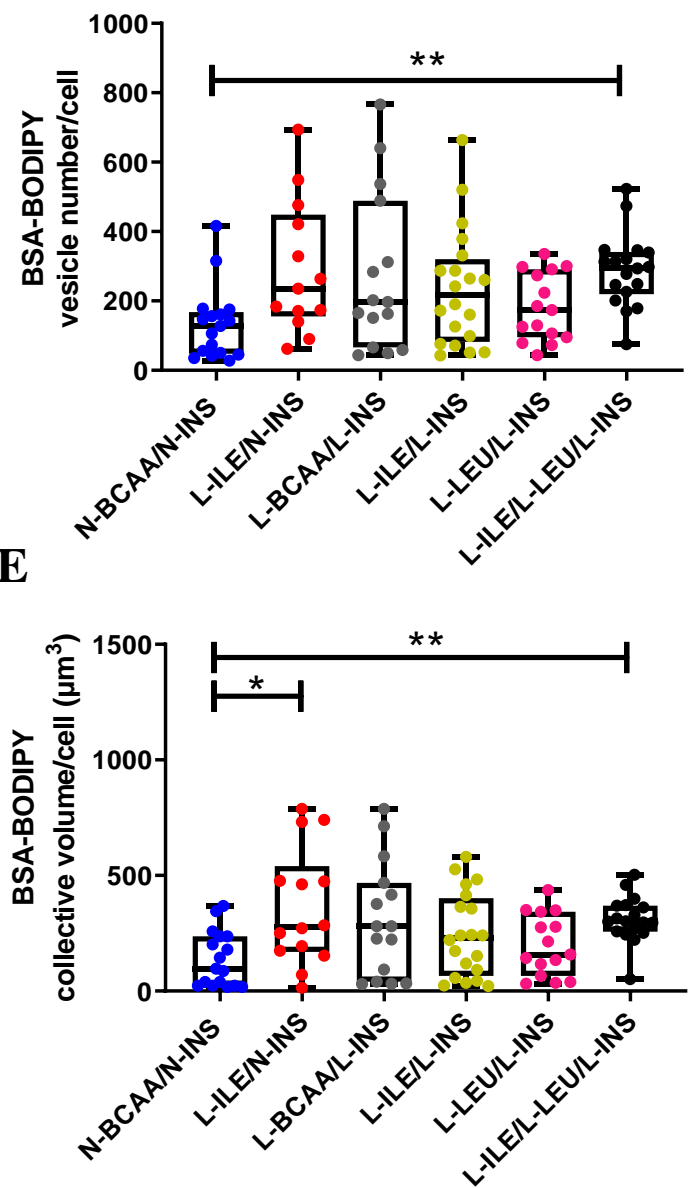
Figure 4

A

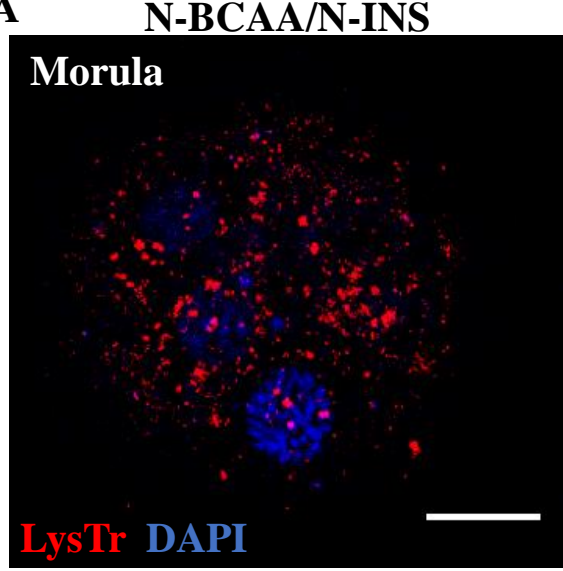

\section{Blastocyst}

B

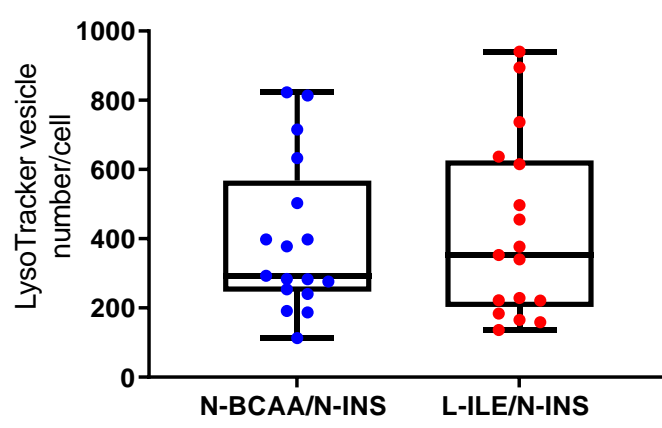

D

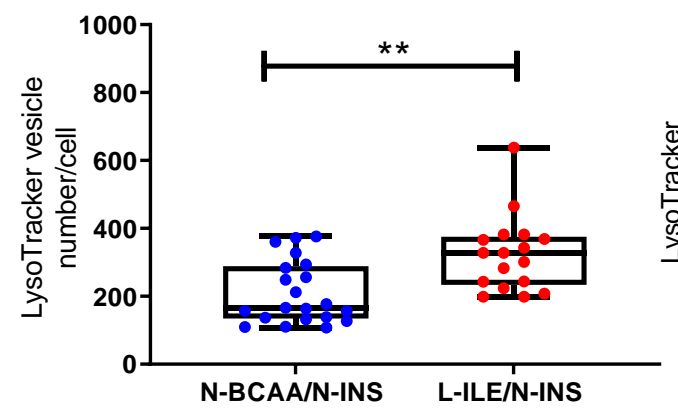

L-ILE/N-INS

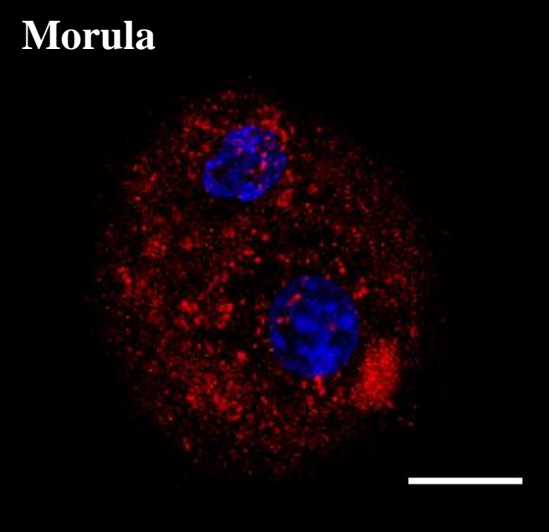

\section{Blastocyst}

C

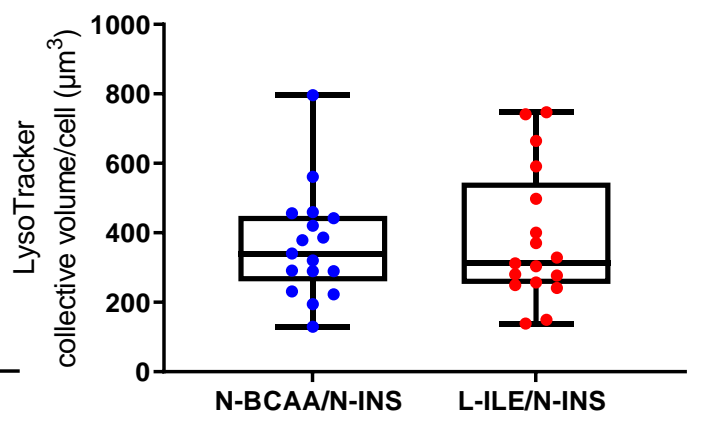

$\mathbf{E}$

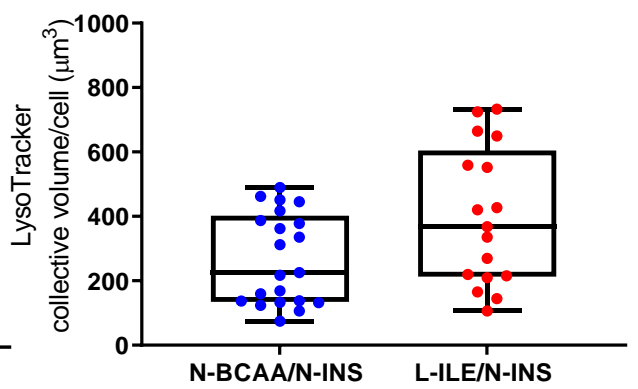


Figure 5

A
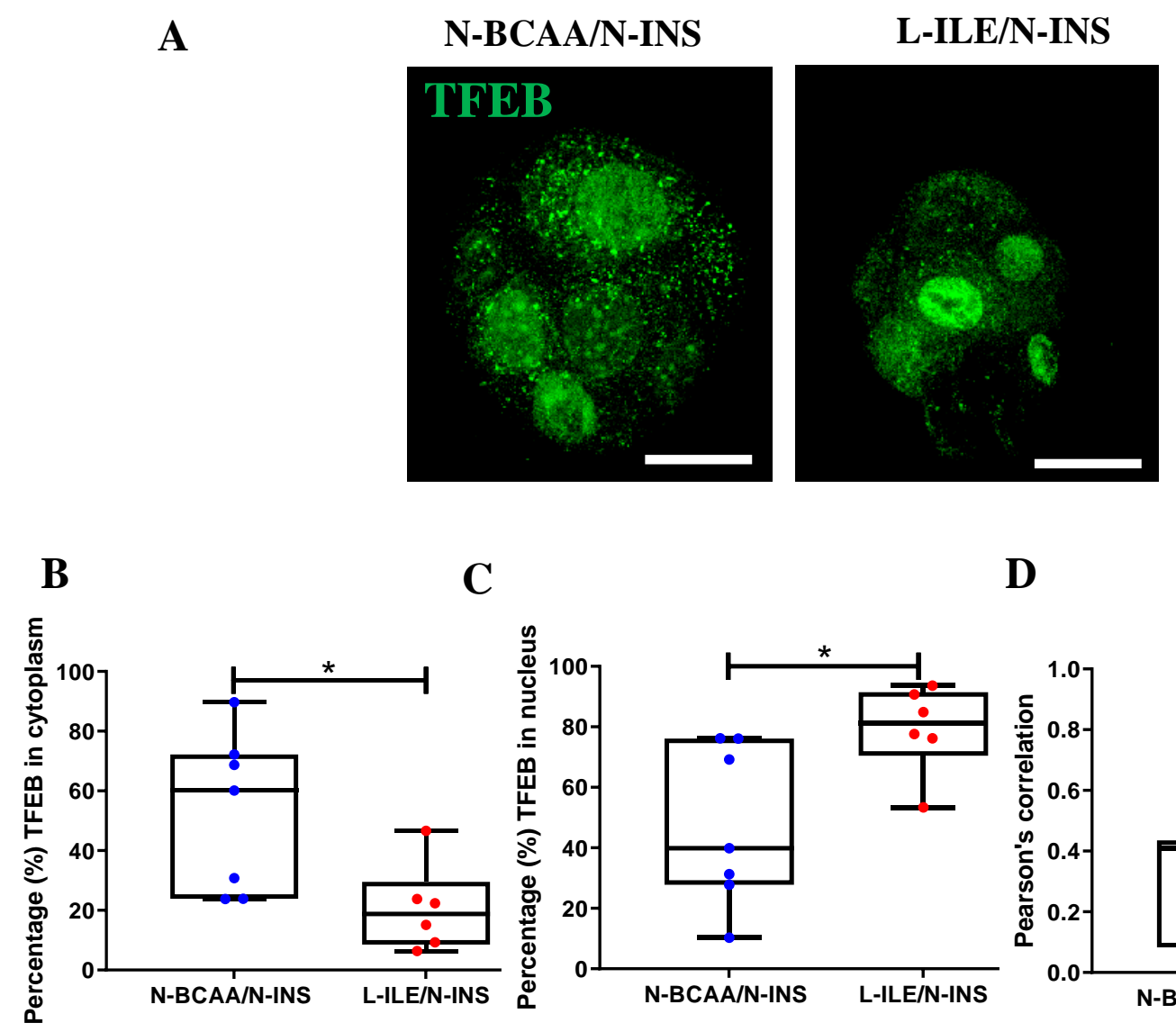

D

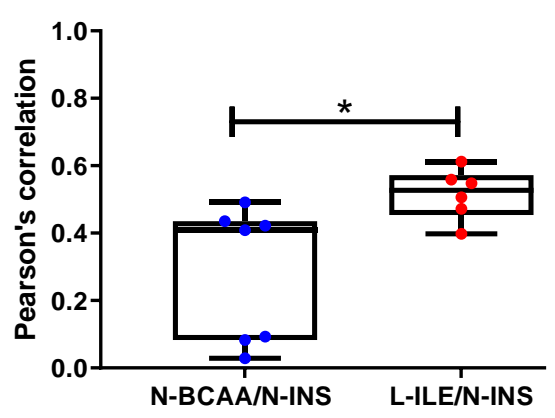


Figure 6

A
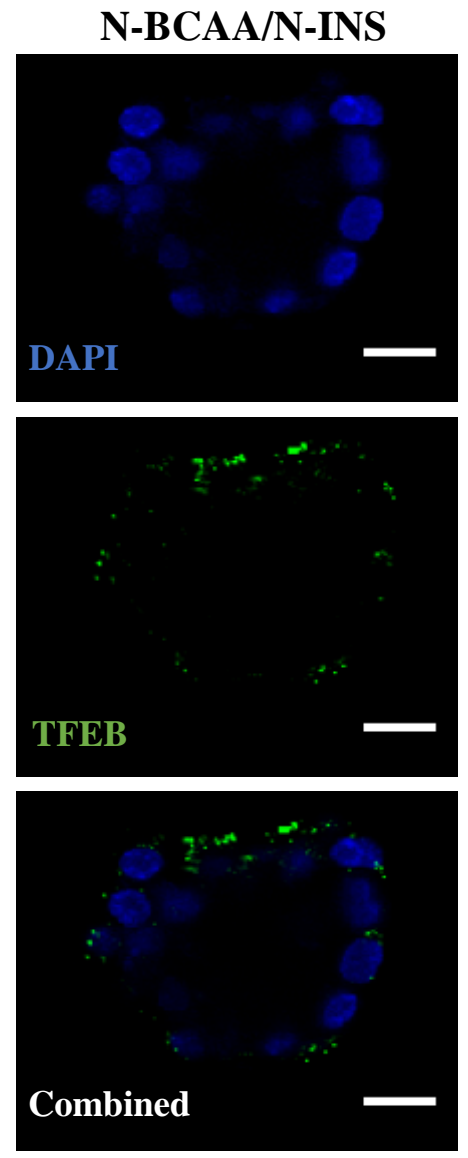

B

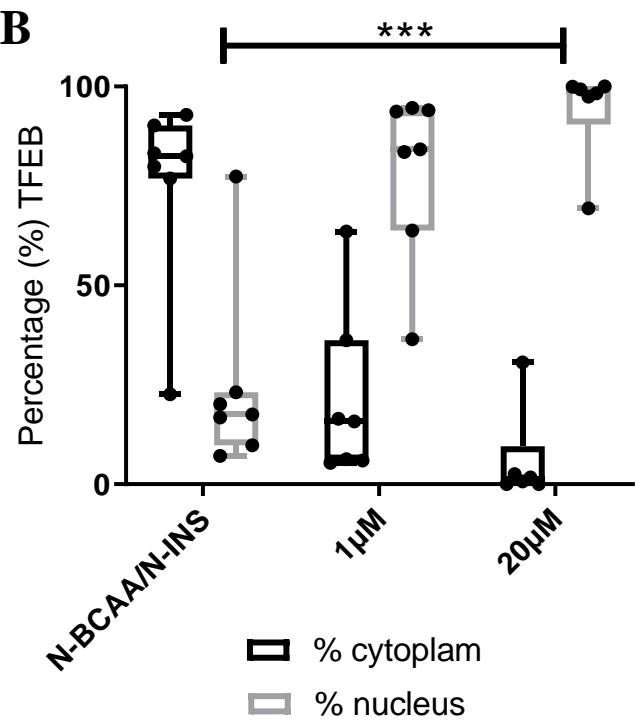

TFEB

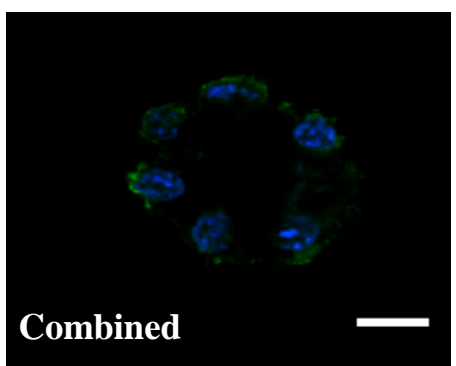

$1 \mu \mathrm{M}$ rapamycin
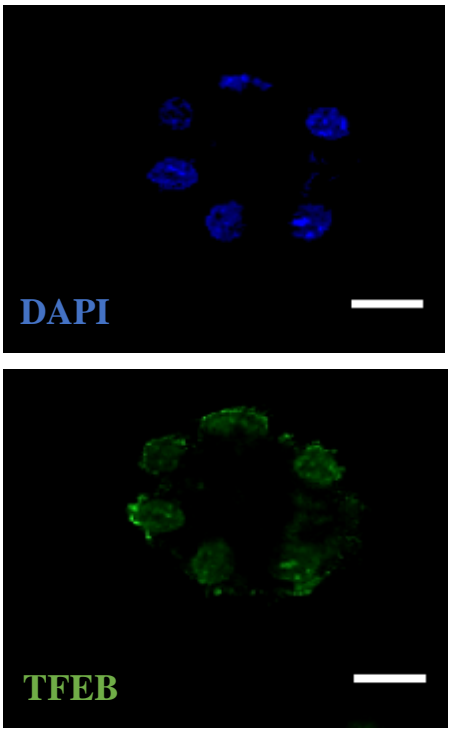

TFEB

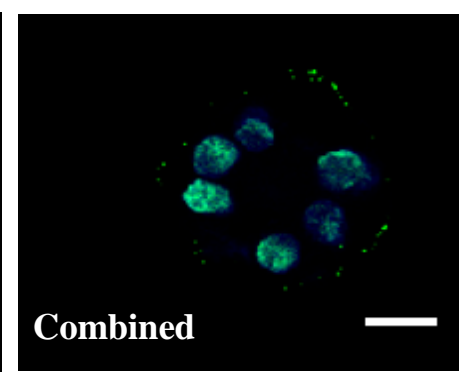

C

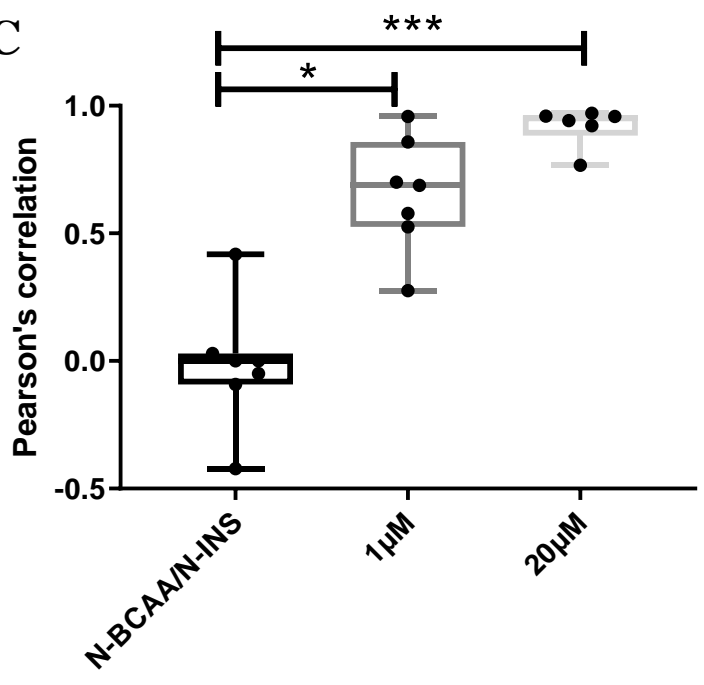

$20 \mu \mathrm{M}$ rapamycin
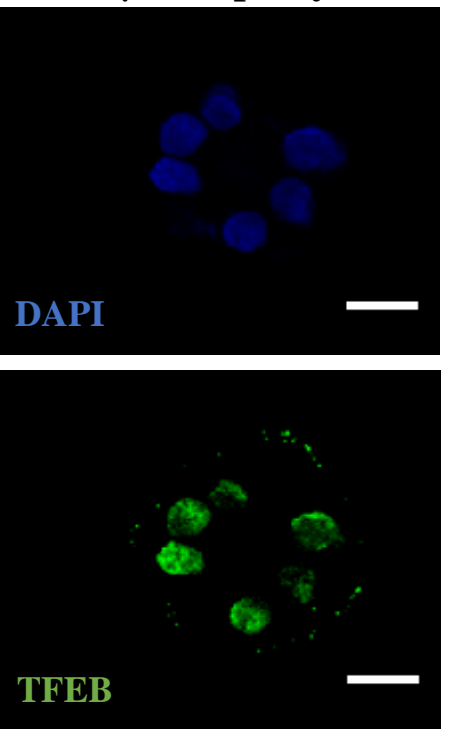


\section{Figure 7}
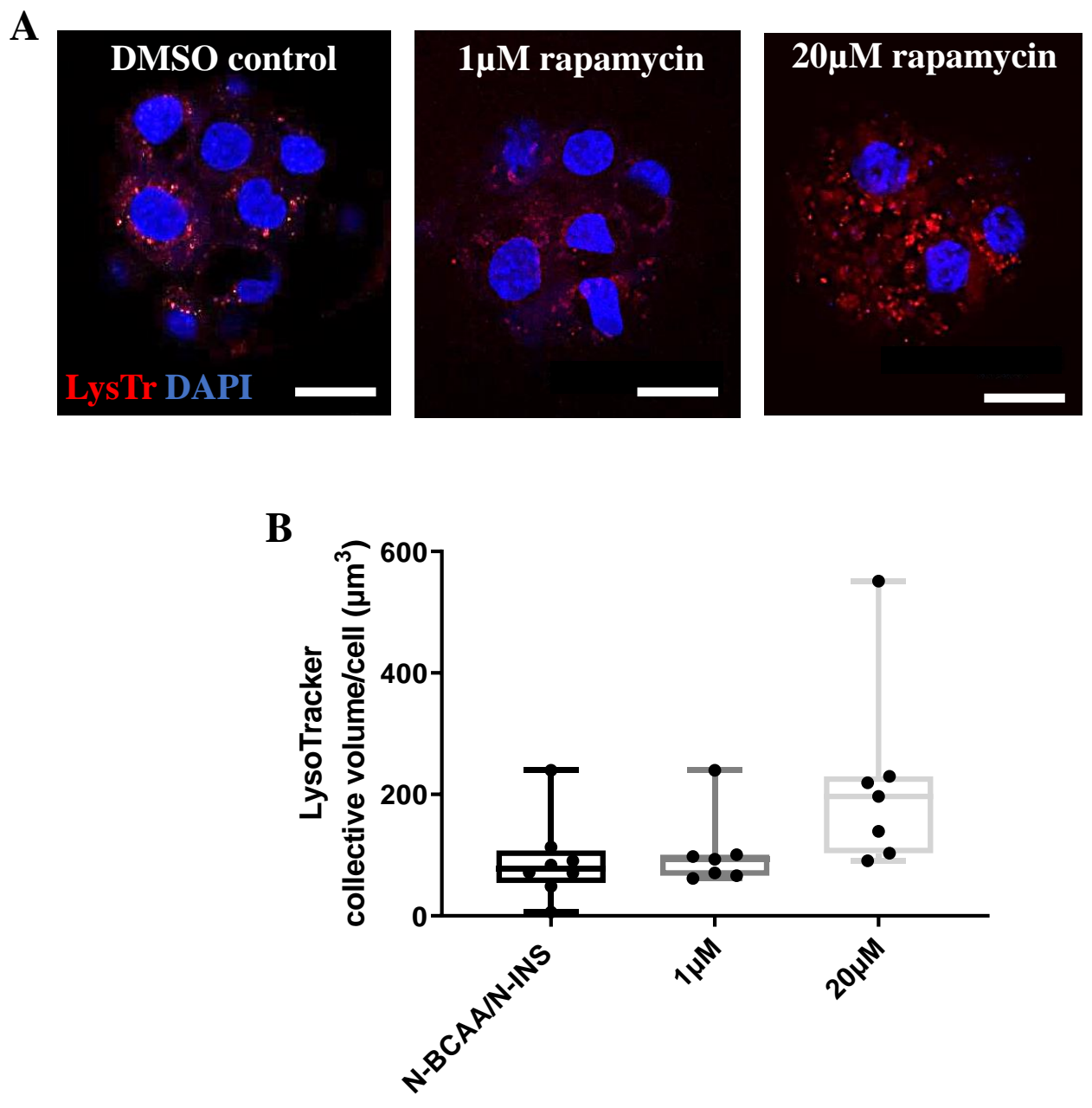


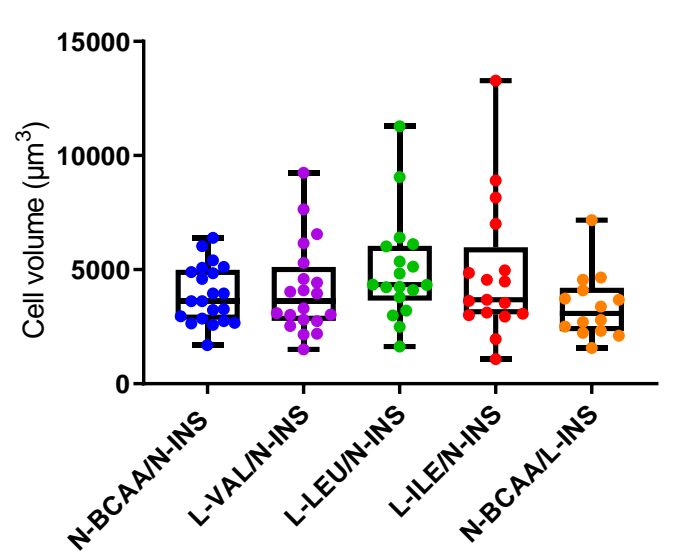

B

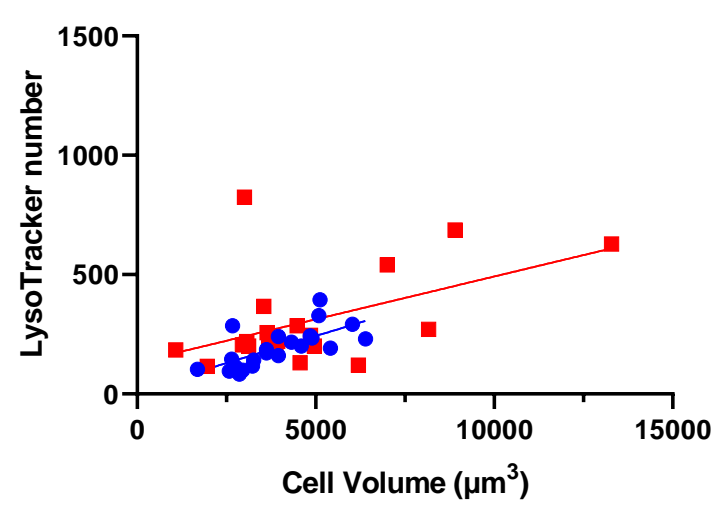

$N$-BCAA/N-INS: $R=0.6839 ; P<0.05$

L-ILE/N-INS: $R=0.4974 ; P<0.05$

D

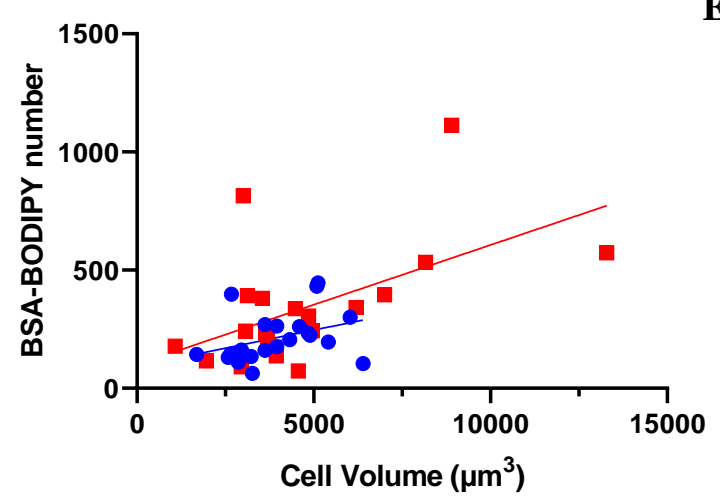

$N-B C A A / N-I N S: R=0.3632 ; P=0.0966$

L-ILE/N-INS: $R=0.5494 ; P<0.05$

F

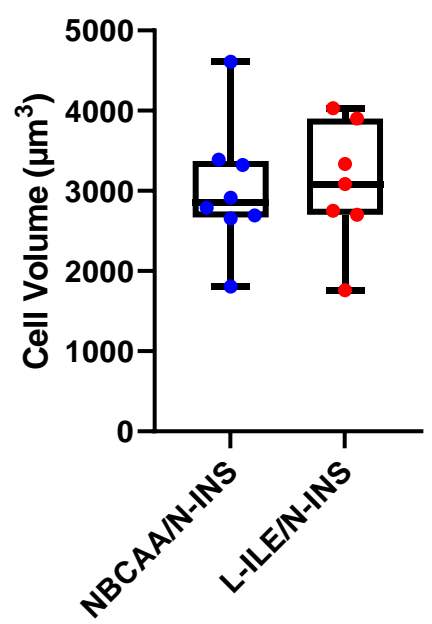

C

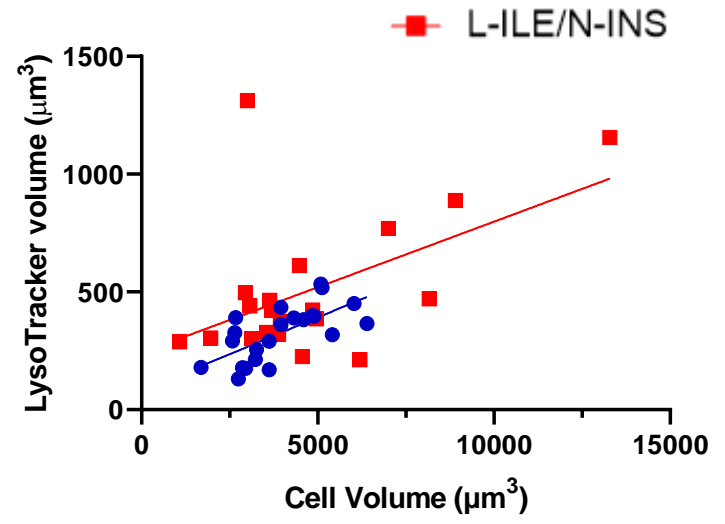

$N-B C A A / N-I N S: R=0.6724 ; P<0.05$

L-ILE/N-INS: $R=0.5160 ; P<0.05$

$\mathbf{E}$

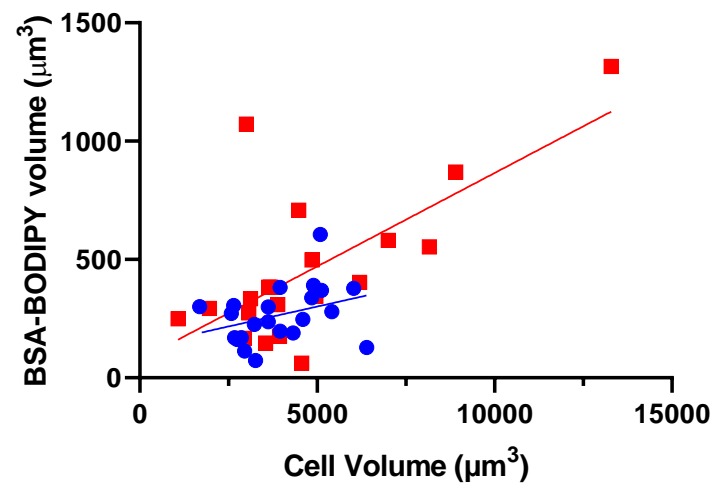

$N$-BCAA/N-INS: $R=0.3504 ; P=0.1099$

L-ILE/N-INS: $R=0.6849 ; P<0.05$

G

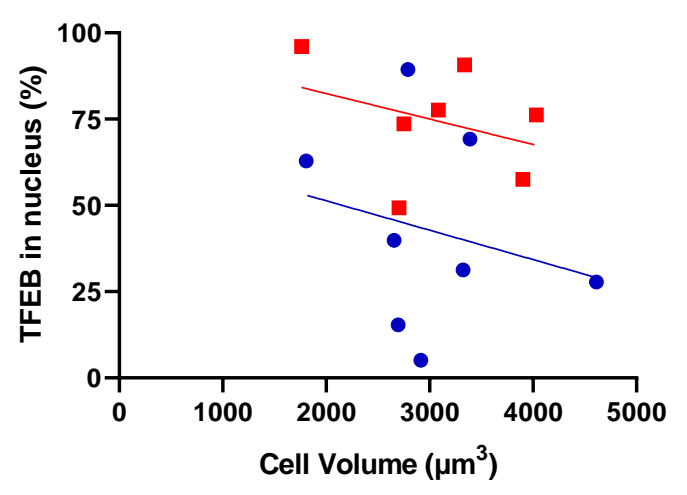

$N$-BCAA/N-INS: $R=-0.2381 ; P=0.5702$

L-ILE/N-INS: $R=-0.3451 ; P=0.4483$ 
Figure 9

A

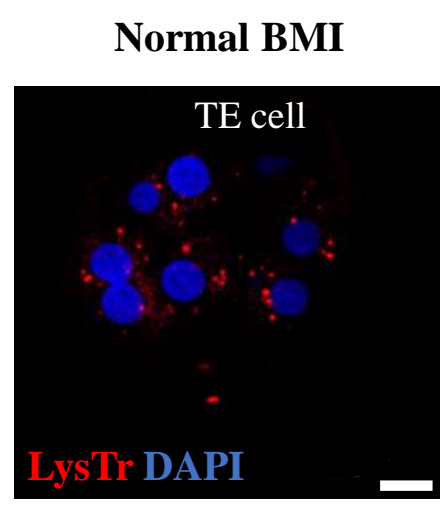

VOLOCITY
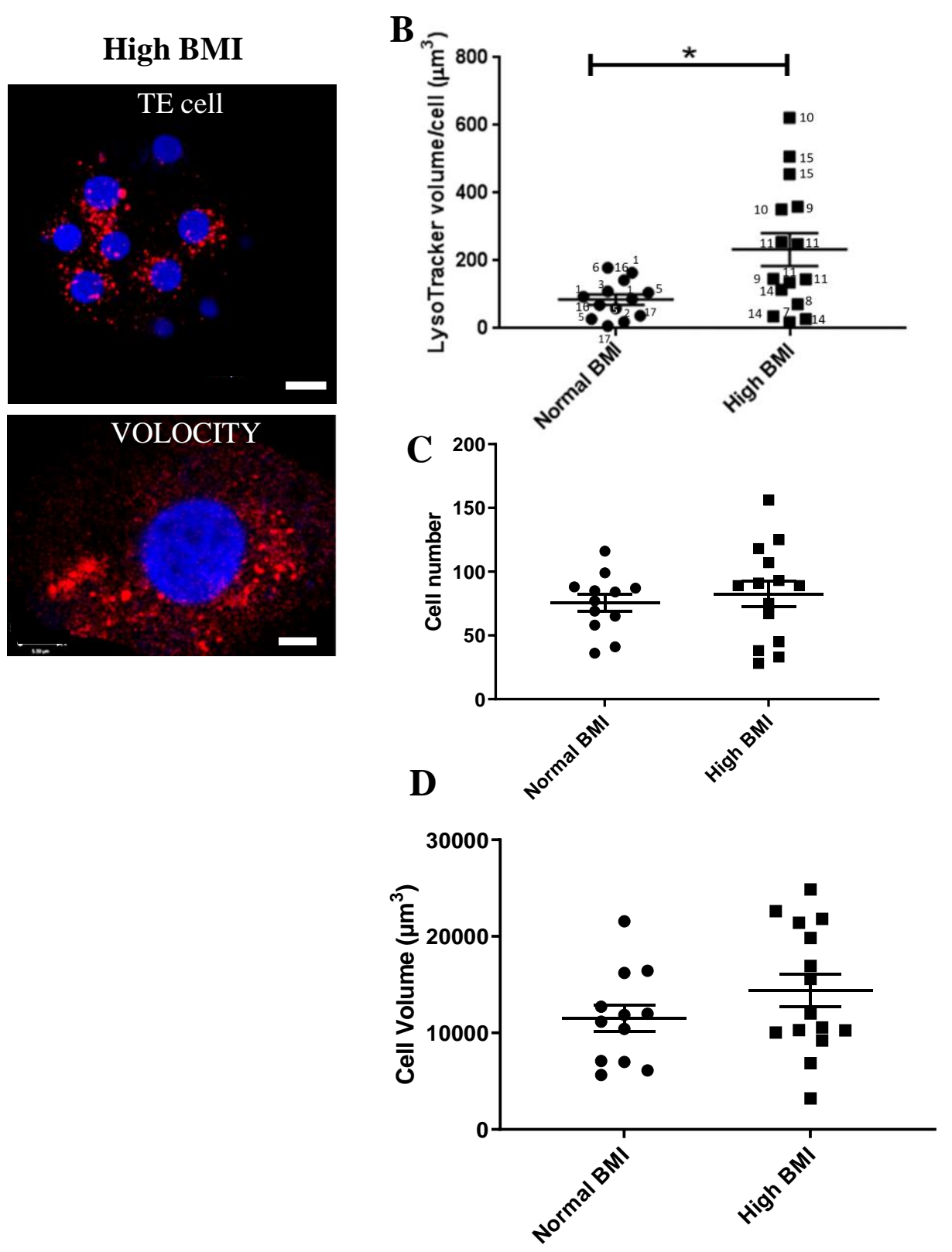
Figure 10

A
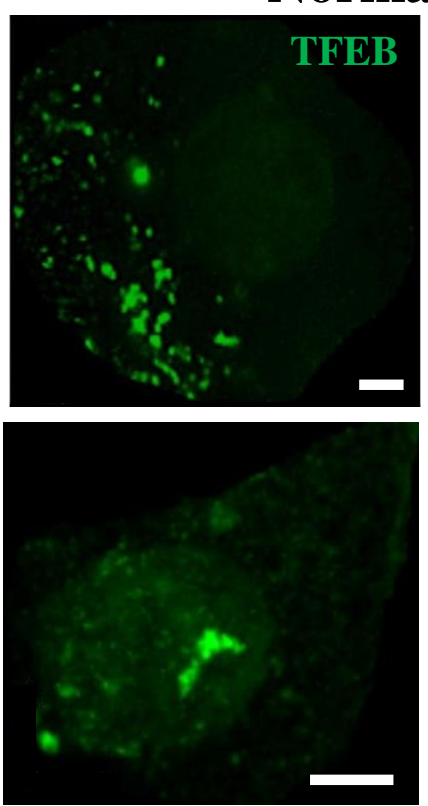

B

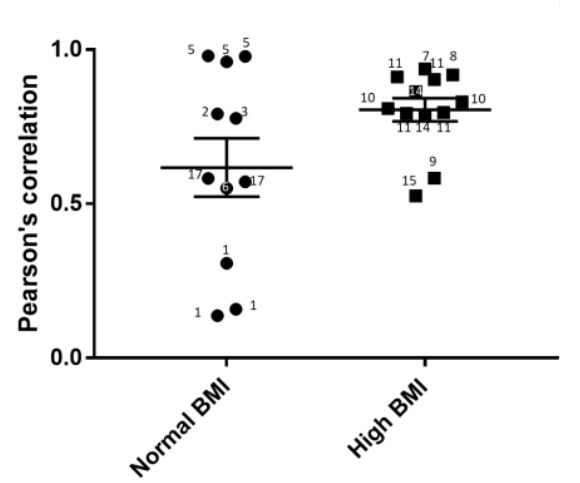

High BMI
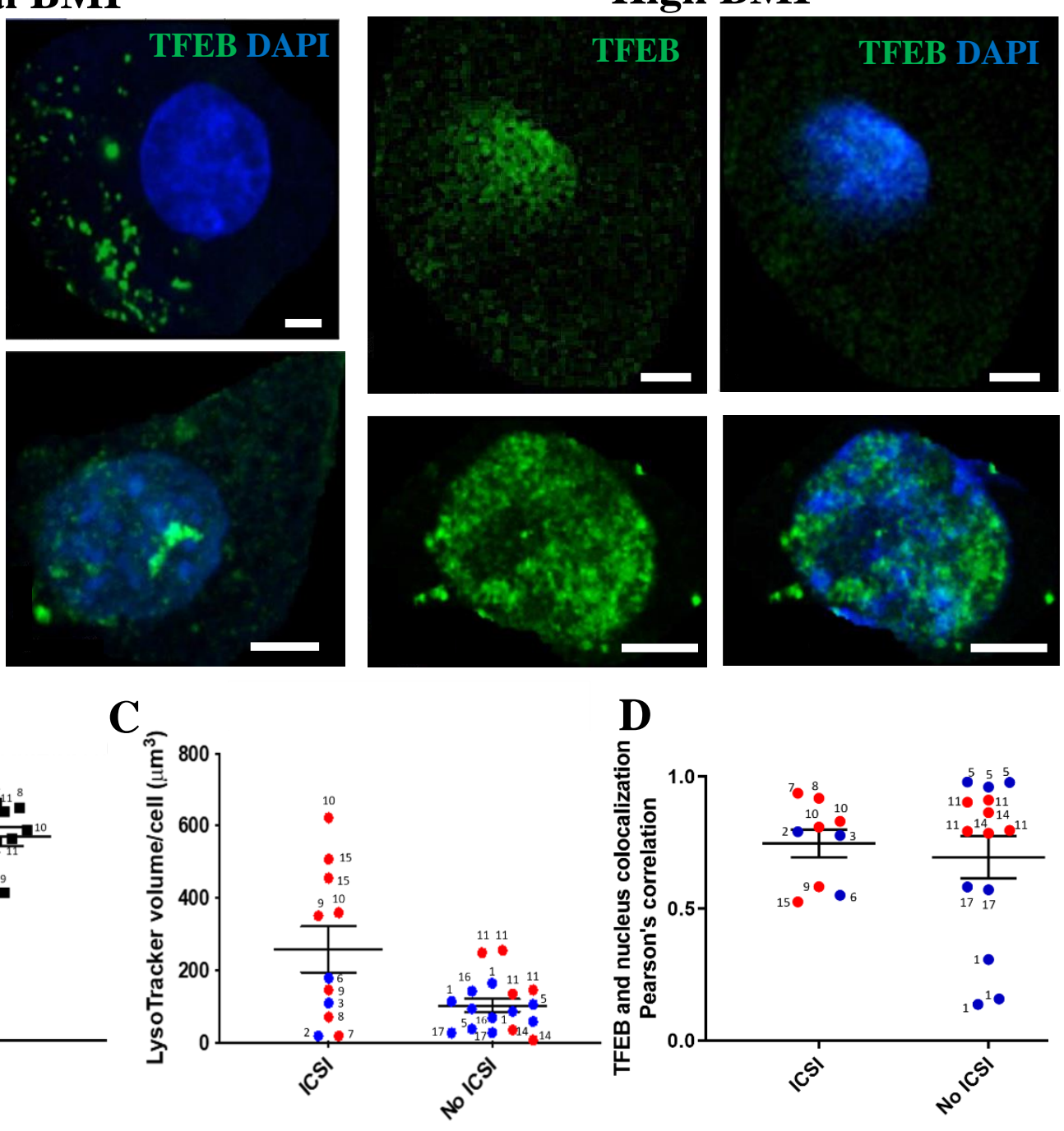


\section{Supplementary Figure 1}

A

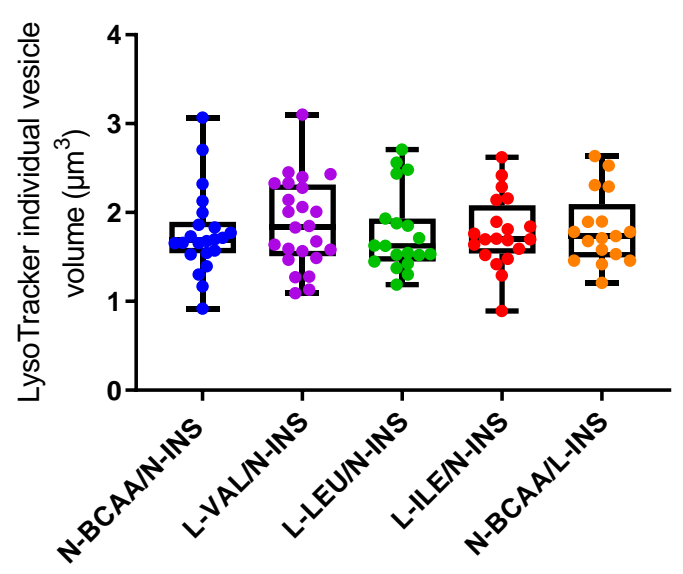

C

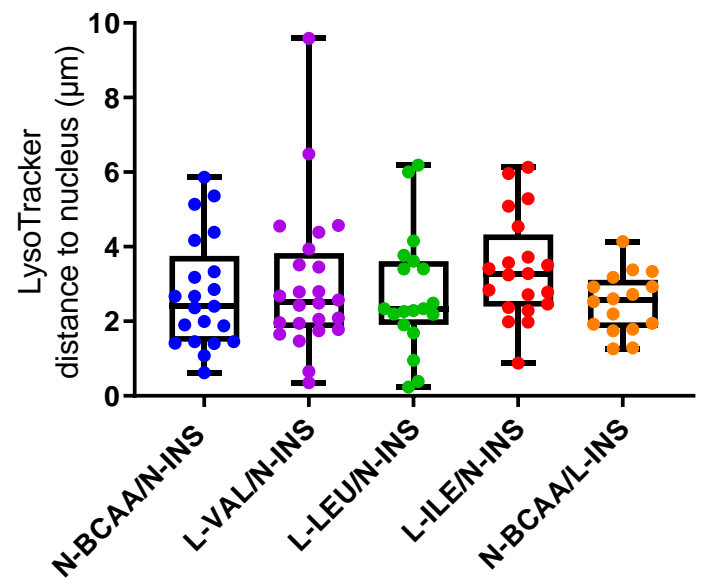

E

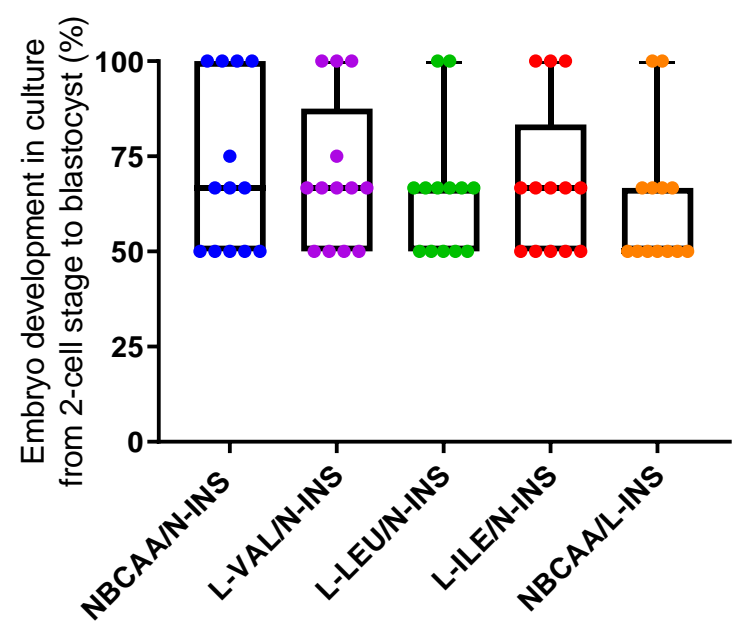

B

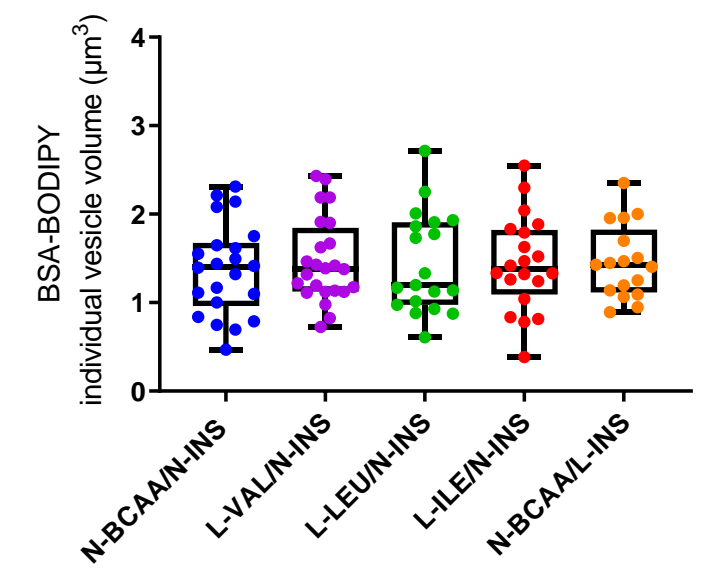

D

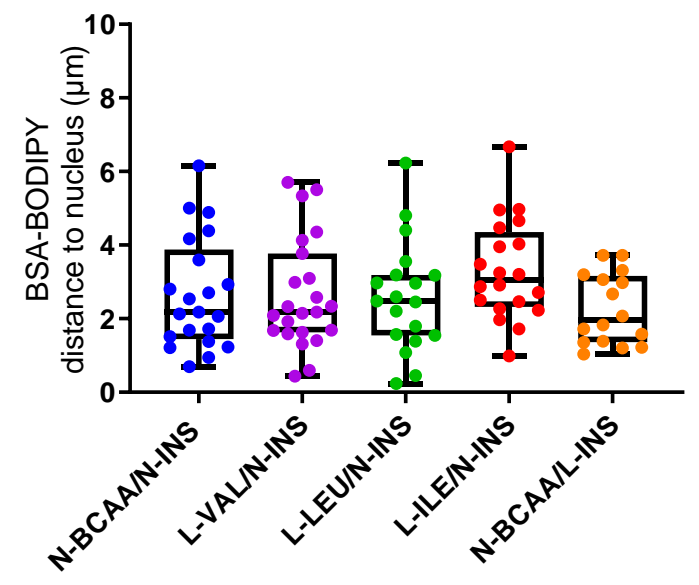

F

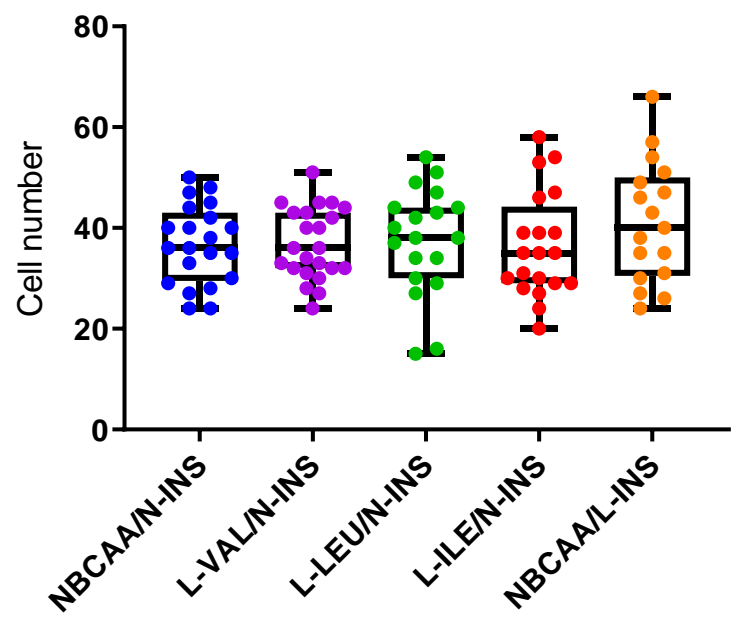


Supplementary Figure 2

A

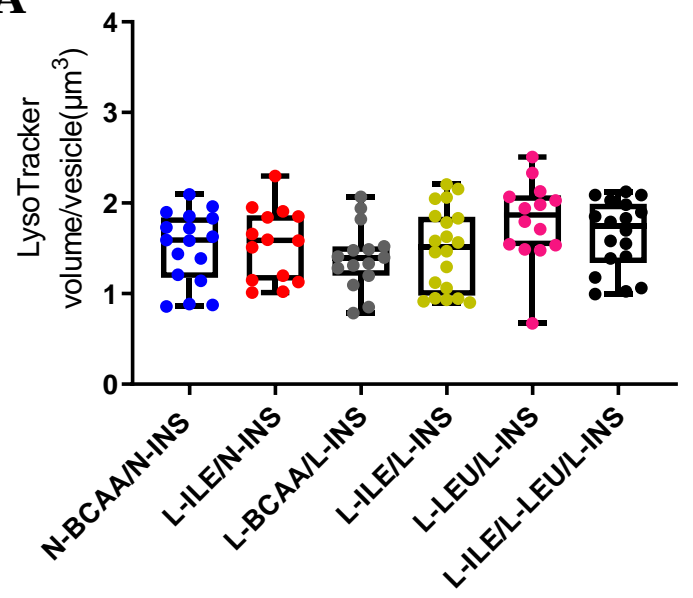

$\mathrm{C}$

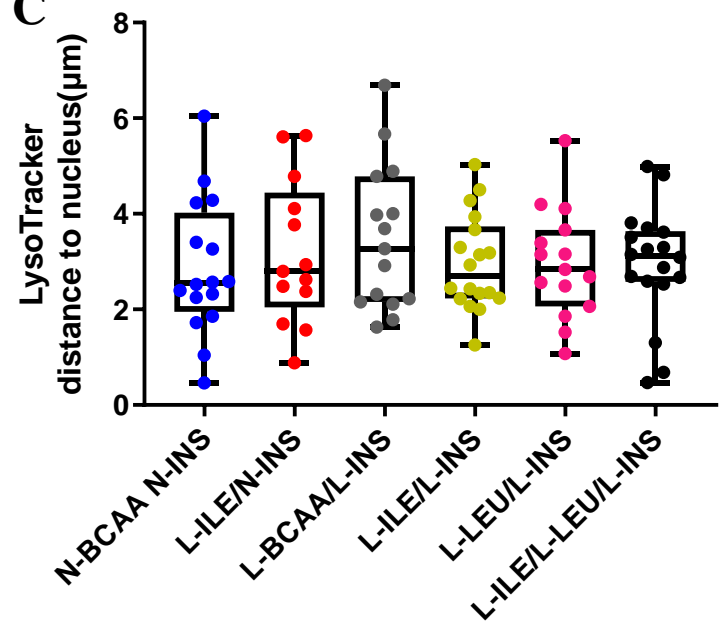

B

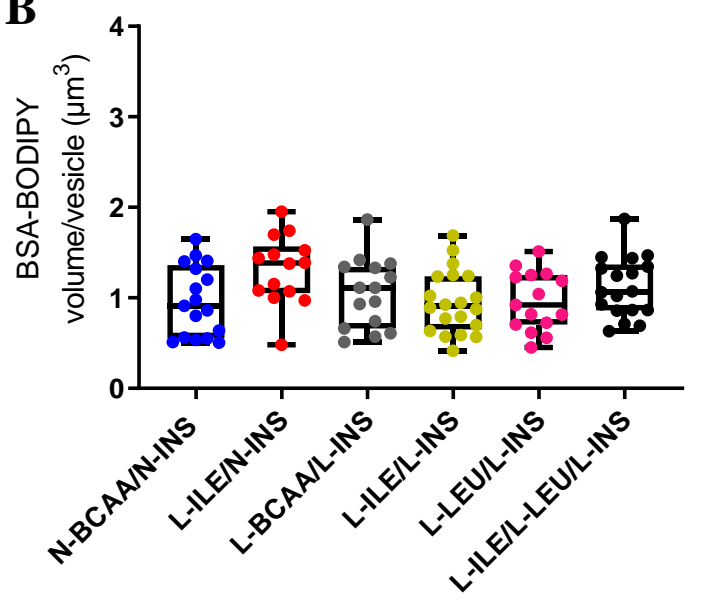

D

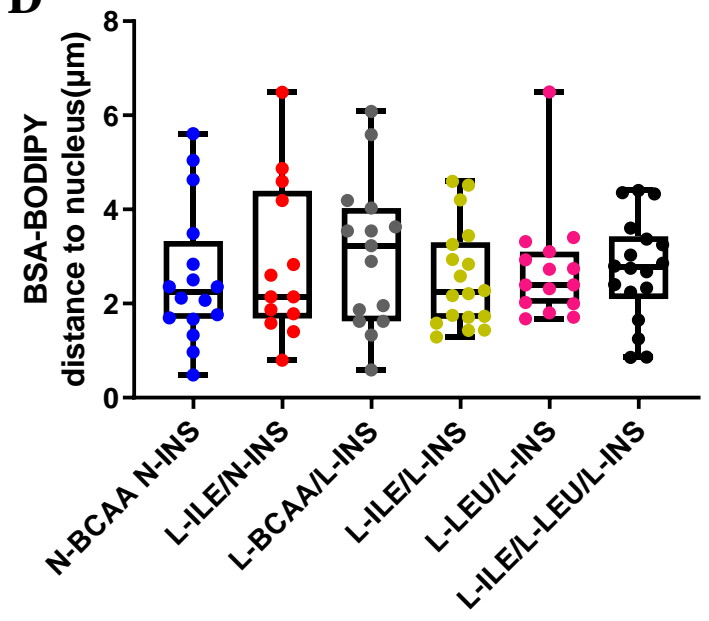

